\title{
Higgsino dark matter in high-scale supersymmetry
}

\author{
Natsumi Nagata ${ }^{a, b}$ and Satoshi Shirai ${ }^{c}$ \\ ${ }^{a}$ William I. Fine Theoretical Physics Institute, School of Physics and Astronomy, \\ University of Minnesota, \\ Minneapolis, MN 55455, U.S.A. \\ ${ }^{b}$ Kavli Institute for the Physics and Mathematics of the Universe (WPI), \\ Todai Institutes for Advanced Study, University of Tokyo, \\ Kashiwa 277-8583, Japan \\ ${ }^{c}$ Deutsches Elektronen-Synchrotron (DESY), \\ 22607 Hamburg, Germany \\ E-mail: nagat006@umn.edu, satoshi.shirai@desy.de
}

ABSTRACT: We study a supersymmetric (SUSY) Standard Model in which a Higgsino is light enough to be dark matter, while the other SUSY particles are much heavier than the weak scale. We carefully treat the effects of heavy SUSY particles to the Higgsino nature, especially taking into account the renormalization effects due to the large hierarchy between the Higgsino and the SUSY breaking scales. Inelastic scattering of the Higgsino dark matter with a nucleus is studied, and the constraints on the scattering by the direct detection experiments are discussed. This gives an upper limit on the new physics scale. Bounds on the dark matter-nucleon elastic scattering, the electric dipole moments, and direct production of Higgsinos, on the other hand, give a lower limit. We show the current status on the limits and discuss the future prospects.

KeYwORDS: Supersymmetry Phenomenology

ARXIV EPRINT: 1410.4549 


\section{Contents}

1 Introduction 1

2 Higgsino mass spectrum $\quad 3$

$\begin{array}{lll}3 & \text { Renormalization of higher dimensional operators } & 7\end{array}$

4 Higgsino dark matter search $\quad 10$

$\begin{array}{ll}4.1 \text { Inelastic scattering } & 10\end{array}$

$\begin{array}{lll}4.2 & \text { Elastic scattering } & 13\end{array}$

$\begin{array}{llr}5 & \text { Electric dipole moments } & 15\end{array}$

$\begin{array}{llr}6 & \text { Collider signals } & 17\end{array}$

$\begin{array}{llr}7 & \text { Summary and discussion } & 18\end{array}$

A Diagonalization of a $2 \times 2$ complex symmetric matrix $\quad 20$

$\begin{array}{ll}\text { B Higgsino gauge interactions in the mass eigenbasis } & 21\end{array}$

$\begin{array}{ll}\text { C Renormalization group equations } & 21\end{array}$

\section{Introduction}

The supersymmetric (SUSY) Standard Model (SSM) is a strong candidate for new physics. The weak-scale SUSY is commonly said to provide a solution to the hierarchy problems, promising frameworks for the grand unification, and the correct amount of dark matter (DM) in the Universe. However, the discovery of the Standard Model (SM)-like Higgs boson with a mass of $\sim 125 \mathrm{GeV}[1,2]$ as well as the absence of new physics seems to imply the SUSY breaking scale is much higher than the weak scale [3-8]. With the SUSY breaking scale larger than $O(10) \mathrm{TeV}$, the observed Higgs mass can be realized [9-14]. The high-scale SUSY scenario may offer an even more precise gauge coupling unification [15] and open up possibilities for the simplest framework of the grand unified theory [16, 17]. With the $R$-parity conservation assumed, it also provides the lightest SUSY particle (LSP) as a DM candidate. In addition, such a high-scale SUSY scenario can greatly relax serious SUSY flavor/CP [18-23] and cosmological problems [24-26]. For these reasons, this framework has been gathering more and more attention these days, especially after the discovery of the Higgs boson [27-32].

In such a high scale SUSY model, however, the weak scale can be realized only with a great extent of fine-tuning. Although the origin of stability of the weak scale is unclear, an 
appealing approach would be utilizing the anthropic principle or environmental selection on multiverse; the $O(100) \mathrm{GeV}$ weak scale is essential for the formulation of complex nuclei [33] that is crucial for the existence of intelligent life, just as in the case of the cosmological constant [34].

This kind of environmental selection may also work on the LSP mass $m_{\text {LSP }}[27,28,35-37]$. A too heavy LSP mass leads to over-abundance of DM in the Universe. To avoid this catastrophe, the LSP mass should be significantly tuned to be around $\mathrm{TeV}$ scale or much heavier than the mass scale of inflaton. If too much abundance of DM is disfavored with the environmental selection [38], a mass region

$$
O(1-10) \mathrm{TeV} \lesssim m_{\mathrm{LSP}} \lesssim \max \left\{10^{2} T_{R}, m_{\mathrm{inf}}\right\}
$$

may be forbidden, where $T_{R}$ is the reheating temperature of the Universe and $m_{\text {inf }}$ is the inflaton mass [39]. The recent report on the search for gravitational waves by BICEP2 [40], for instance, may indicate $m_{\mathrm{inf}} \sim 10^{13} \mathrm{GeV}$, though the interpretation of the result is controversial [41]. Further, $T_{R} \simeq 10^{9} \mathrm{GeV}$ is necessary condition for the successful thermal leptogenesis [42]. Anyway, we expect large hierarchy between the electroweak scale and the energy scale of $T_{R}$. To evade the above unacceptable window, an environmental selection may work to let the LSP mass remain TeV scale, which results in a considerable fine-tuning for the LSP mass parameter. In this case, the "lonely LSP" scenario, in which only the LSP is around TeV scale and the other SUSY particles are much heavier, can be realized.

Even without such an anthropic viewpoint, the "lonely LSP" scenario can be achieved for some dynamical reasons. For example, if a certain symmetry forbids the tree-level LSP mass and it is generated only by radiative corrections, the LSP mass will be much suppressed compared to those of the other SUSY particles. Among the minimal SSM (MSSM) particles, an experimentally viable candidate for the LSP DM is a Higgsino or a Wino. Although a Bino or a gravitino LSP would be possible, its abundance strongly depends on the high-energy model and tends to be produced too much. The Wino DM case has been widely considered so far $[43,44]$ since it is motivated by the anomaly mediation $[45,46]$, and their phenomenology is thoroughly discussed in previous works [28, 47-59]. The Higgsino LSP is also viable, for its mass can be suppressed by some symmetries such as the Peccei-Quinn symmetry [60] or the $R$-symmetry. In this paper, we focus on this Higgsino LSP case. Indeed, the Higgsino mass with a mass of $\sim 1 \mathrm{TeV}$ can explain the observed DM density [61], while the environmental selection arguments may suggest that the Higgsino LSP has a mass of $\mathcal{O}(100) \mathrm{GeV}$ (unless it is much heavier than the inflation scale). This mass region is the target of the present study. For the former arguments, see, e.g., refs. [62-66].

The "lonely Higgsino" actually cannot be completely lonely, for a pure Higgsino DM has been already excluded by the DM direct detection experiments. Tiny amount of mixing among the Higgsino and gauginos is required to avoid the constraints, which gives an upperbound on the SUSY breaking scale. It turns out that the scale is much larger than the $\mathrm{TeV}$ scale. Such a large mass hierarchy induces large quantum corrections. Thus, to study the properties of the Higgsino DM precisely, we need to take the effects into account. 
In this work, we revisit the phenomenology of the Higgsino LSP considering the renormalization corrections due to the large hierarchy between the Higgsino mass and the SUSY breaking scales. These corrections affect the mass splitting between the neutral Higgsinos, which are important to discuss the constraints on it coming from the inelastic scatterings of the Higgsino DM with a nucleon. We will study these constraints in the case of the Higgsino DM in detail and by using the results derive an upper limit on the gaugino mass scale. The mass splitting depends on new CP-phases appearing in the gaugino and Higgsino masses as well, and the phases can be probed by means of the electric dipole moments (EDMs). We will discuss the interplay between the bounds from the EDM measurements and the DM direct detection experiments. The elastic scattering of the Higgsino DM with a nucleon, as well as the direct production of Higgsinos in colliders, is also discussed with their future prospects. We will find that the constraints from the measurements of the above quantities are complementary to each other. By considering them altogether, we may probe the nature of the Higgsino DM and the signature of high-scale physics in future experiments, which enables us to gain an insight on the SSM.

The organization of this paper is as follows. In the next section, we study the mass spectrum of Higgsinos and new physics effects on it. The effects are expressed in terms of the dimension-five effective operators. Then, in section 3, we present the renormalization group equations (RGEs) for the operators as well as their matching conditions, and study the renormalization effects on them. By using the results, we discuss the constraints on the Higgsino DM scenario from the direct detection experiments, the measurements of the EDMs, and the Higgsino searches in colliders in section 4, section 5, and section 6, respectively. Section 7 is devoted to summary of the results and discussion.

\section{$2 \quad$ Higgsino mass spectrum}

To begin with, we give a brief review on the mass spectrum of Higgsinos in the presence of small mixing with gauginos whose masses are assumed to be much heavier than the Higgsino masses. The dominant mixing effects are included in the dimension-five effective operators shown below. Their coefficients as well as the renormalization effects on them are evaluated in the subsequent section.

In the MSSM, the mass term for Higgsinos $\widetilde{H}_{u}$ and $\widetilde{H}_{d}$ is given as

$$
\mathcal{L}_{\text {Higgsino mass }}=-\mu \epsilon^{\alpha \beta}\left(\widetilde{H}_{u}\right)_{\alpha}\left(\widetilde{H}_{d}\right)_{\beta}+\text { h.c. },
$$

where $\alpha$ and $\beta$ are the $\mathrm{SU}(2)_{L}$ indices, $\epsilon^{\alpha \beta}$ is an antisymmetric tensor with $\epsilon^{12}=-\epsilon^{21}=+1$, and

$$
\widetilde{H}_{u}=\left(\begin{array}{c}
\widetilde{H}_{u}^{+} \\
\widetilde{H}_{u}^{0}
\end{array}\right), \quad \widetilde{H}_{d}=\left(\begin{array}{c}
\widetilde{H}_{d}^{0} \\
\widetilde{H}_{d}^{-}
\end{array}\right) .
$$

As one can see, $\widetilde{H}_{u}$ and $\widetilde{H}_{d}$ form a Dirac fermion. Thus, there is a U(1) symmetry under which $\widetilde{H}_{u}$ and $\widetilde{H}_{d}$ are oppositely charged. If there exist operators which break the U(1) symmetry, however, the Dirac fermion is divided into a pair of Majorana fermions. Up to 
dimension-five, such operators are given as ${ }^{1}$

$$
\mathcal{L}_{\text {eff }}=\sum_{i=1,2} c_{i} \mathcal{O}_{i}+\text { h.c. }
$$

where

$$
\begin{aligned}
& \mathcal{O}_{1} \equiv\left(H^{\dagger}\right)^{\alpha}\left(\widetilde{H}_{u}\right)_{\alpha}\left(H^{\dagger}\right)^{\beta}\left(\widetilde{H}_{u}\right)_{\beta}, \\
& \mathcal{O}_{2} \equiv \epsilon^{\alpha \beta} \epsilon^{\gamma \delta}(H)_{\alpha}\left(\widetilde{H}_{d}\right)_{\beta}(H)_{\gamma}\left(\widetilde{H}_{d}\right)_{\delta},
\end{aligned}
$$

and

$$
H=\left(\begin{array}{c}
H^{+} \\
H^{0}
\end{array}\right)
$$

denotes the SM Higgs field. These operators give rise to the mass splitting between the neutral components of the Higgsinos. We also have the dimension-five operators that do not violate the $\mathrm{U}(1)$ symmetry:

$$
\mathcal{L}_{\text {eff }}=\sum_{i=1,2} d_{i} \widetilde{\mathcal{O}}_{i}+\text { h.c. }
$$

with

$$
\begin{gathered}
\widetilde{\mathcal{O}}_{1} \equiv \epsilon^{\beta \gamma}\left(H^{\dagger}\right)^{\alpha}\left(\widetilde{H}_{u}\right)_{\alpha}(H)_{\beta}\left(\widetilde{H}_{d}\right)_{\gamma}, \\
\widetilde{\mathcal{O}}_{2} \equiv \epsilon^{\beta \gamma}\left(H^{\dagger}\right)^{\alpha}\left(\widetilde{H}_{d}\right)_{\alpha}(H)_{\beta}\left(\widetilde{H}_{u}\right)_{\gamma} .
\end{gathered}
$$

These two operators yield the mass difference between the neutral and charged components. Note that by using the Fierz identities one can easily show that

$$
\epsilon^{\alpha \beta}|H|^{2}\left(\widetilde{H}_{u}\right)_{\alpha}\left(\widetilde{H}_{d}\right)_{\beta}=\widetilde{\mathcal{O}}_{1}-\widetilde{\mathcal{O}}_{2} .
$$

Therefore, the operators $\mathcal{O}_{i}$ and $\widetilde{\mathcal{O}}_{i}$ exhaust the dimension-five operators which consist of the Higgsinos and the Higgs field and are allowed by the gauge and Lorentz symmetries.

Let us examine the mass differences induced by the above operators. After the electroweak symmetry breaking, the mass matrix for the neutral components is given by

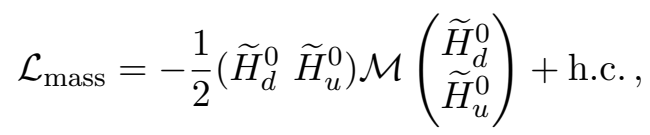

with

$$
\mathcal{M}=\left(\begin{array}{cc}
-v^{2}(|\mu|) c_{2}(|\mu|) & -\bar{\mu} \\
-\bar{\mu} & -v^{2}(|\mu|) c_{1}(|\mu|)
\end{array}\right),
$$

where $v \simeq 246 \mathrm{GeV}$ is the Higgs vacuum expectation value (VEV) and $\bar{\mu} \equiv \mu-$ $v^{2}(|\mu|) d_{1}(|\mu|) / 2$. The parameters and the Wilson coefficients in the mass matrix are renormalized at the scale of $|\mu|$. We omit the argument in the following discussion, for brevity. The mass matrix $\mathcal{M}$ is diagonalized ${ }^{2}$ by using an unitary matrix $N$ as

$$
N^{*} \mathcal{M} N^{\dagger}=\left(\begin{array}{cc}
m_{1} & 0 \\
0 & m_{2}
\end{array}\right),
$$

${ }^{1}$ Notice that operators like $\epsilon^{\alpha \beta} \epsilon^{\gamma \delta}(H)_{\alpha}(H)_{\beta}\left(\widetilde{H}_{d}\right)_{\gamma}\left(\widetilde{H}_{d}\right)_{\delta}$ vanish since the Higgs field is bosonic.

${ }^{2}$ In appendix A, we summarize formulae for the diagonalization of a $2 \times 2$ complex symmetric matrix. 
and the resultant masses $m_{1}$ and $m_{2}$ are given as

$$
\begin{aligned}
& m_{1} \simeq|\bar{\mu}|-\frac{\left|\mu^{*} c_{1}+\mu c_{2}^{*}\right|}{2|\mu|} v^{2}, \\
& m_{2} \simeq|\bar{\mu}|+\frac{\left|\mu^{*} c_{1}+\mu c_{2}^{*}\right|}{2|\mu|} v^{2},
\end{aligned}
$$

where we keep the $\mathcal{O}\left(v^{2}\right)$ terms. In this case, the mass difference between the neutral components is found to be $\mathrm{b}^{3}$

$$
\Delta m \equiv m_{2}-m_{1} \simeq \frac{\left|\mu^{*} c_{1}+\mu c_{2}^{*}\right|}{|\mu|} v^{2} .
$$

The expression indicates that the mass difference depends on the phases in the $\mu$-term and the Wilson coefficients $c_{1}$ and $c_{2}$. The unitary matrix $N$ is evaluated as

$$
N=e^{\frac{i}{2} \phi_{\mu}}\left(\begin{array}{ccc}
e^{-\frac{i}{2}(\phi+\alpha)} \cos \theta & -e^{\frac{i}{2}(\phi-\alpha)} \sin \theta \\
i e^{-\frac{i}{2}(\phi+\beta)} \sin \theta & i e^{\frac{i}{2}(\phi-\beta)} \cos \theta
\end{array}\right),
$$

with

$$
\tan \theta \simeq 1+\frac{\left(\left|c_{2}\right|^{2}-\left|c_{1}\right|^{2}\right) v^{2}}{2\left|\mu^{*} c_{1}+\mu c_{2}^{*}\right|}
$$

and

$$
\begin{aligned}
\phi & =\arg \left(\bar{\mu}^{*} c_{1}+\bar{\mu} c_{2}^{*}\right), & \phi_{\mu} & =\arg (\mu), \\
\alpha & =\frac{v^{2}}{2} \operatorname{Im}\left(\frac{d_{1}+2 c_{2} e^{i \phi}}{\mu}\right), & \beta & =\frac{v^{2}}{2} \operatorname{Im}\left(\frac{d_{1}-2 c_{1} e^{-i \phi}}{\mu}\right) .
\end{aligned}
$$

Again, we remain the terms up to $\mathcal{O}\left(v^{2}\right)$. By using the unitary matrix, the mass eigenstates are written as follows:

$$
\left(\begin{array}{l}
\widetilde{\chi}_{1}^{0} \\
\widetilde{\chi}_{2}^{0}
\end{array}\right)=N\left(\begin{array}{c}
\widetilde{H}_{d}^{0} \\
\widetilde{H}_{u}^{0}
\end{array}\right) .
$$

Here, $\widetilde{\chi}_{1}^{0}$ and $\widetilde{\chi}_{2}^{0}$ are the mass eigenstates corresponding to $m_{1}$ and $m_{2}$, respectively.

The mass term of the charged Higgsino is, on the other hand, given by

$$
\mathcal{L}_{\text {mass }}=-\left(\mu+\frac{v^{2}}{2} d_{2}\right) \widetilde{H}_{u}^{+} \widetilde{H}_{d}^{-}+\text {h.c. . }
$$

Through the field redefinition, we can write the mass term with the mass eigenstate $\widetilde{\chi}^{+}$as

$$
\mathcal{L}_{\text {mass }}=-m_{\tilde{\chi}^{ \pm}} \overline{\tilde{\chi}^{+}} \tilde{\chi}^{+}+\text {h.c. } .
$$

Here, $\widetilde{\chi}^{+}$is a four-component Dirac fermion defined by

$$
\widetilde{\chi}^{+} \equiv\left(\begin{array}{c}
e^{\frac{i}{2}\left(\phi_{\mu}+\gamma\right)} \widetilde{H}_{u}^{+} \\
e^{-\frac{i}{2}\left(\phi_{\mu}+\gamma\right)}\left(\widetilde{H}_{d}^{-}\right)^{\dagger}
\end{array}\right),
$$

\footnotetext{
${ }^{3}$ The result differs from that presented in ref. [67].
} 
with

$$
m_{\widetilde{\chi}^{ \pm}}=\left|\mu+\frac{v^{2}}{2} d_{2}\right|, \quad \gamma=\frac{v^{2}}{2} \operatorname{Im}\left(\frac{d_{2}}{\mu}\right) .
$$

From the mass parameters obtained above, one can easily find that the higher-dimensional operators also contribute to the mass difference between charged Higgsino and the Higgsino DM. The contribution $\left.\Delta m_{+}\right|_{\text {tree }}$ is given by

$$
\left.\Delta m_{+}\right|_{\text {tree }} \simeq \frac{v^{2}}{2}\left[|\mu| \operatorname{Re}\left(\frac{d_{1}+d_{2}}{\mu}\right)+\frac{\left|\mu^{*} c_{1}+\mu c_{2}^{*}\right|}{|\mu|}\right] .
$$

In addition, it is known that radiative corrections by the electroweak gauge bosons induce the neutral-charged Higgsino mass difference. At one-loop level, the contribution is expressed as

$$
\left.\Delta m_{+}\right|_{\mathrm{rad}}=\frac{\alpha_{2}}{4 \pi} m_{\widetilde{\chi}^{ \pm}} \sin ^{2} \theta_{W} f\left(\frac{m_{Z}}{m_{\tilde{\chi}^{ \pm}}}\right),
$$

where $\alpha_{2} \equiv g^{2} /(4 \pi)$ with $g$ the $\mathrm{SU}(2)_{L}$ gauge coupling constant, $\theta_{W}$ is the weak mixing angle, and $m_{Z}$ is the mass of $Z$ boson. The function $f(x)$ is given by ${ }^{4}$

$$
f(x)=2 \int_{0}^{1} d t(1+t) \ln \left(1+\frac{x^{2}(1-t)}{t^{2}}\right) .
$$

Especially, in the limit of $x \rightarrow 0$,

$$
f(x) \simeq 2 \pi x-3 x^{2}+\ldots,
$$

and thus eq. (2.24) is approximated by

$$
\left.\Delta m_{+}\right|_{\mathrm{rad}} \simeq \frac{1}{2} \alpha_{2} m_{Z} \sin ^{2} \theta_{W}\left(1-\frac{3 m_{Z}}{2 \pi m_{\widetilde{\chi}^{ \pm}}}\right) .
$$

In figure 1, we show the radiative corrections to the neutral-charged Higgsino mass difference $\left.\Delta m_{+}\right|_{\text {rad }}$ as a function of the Higgsino mass parameter $|\mu|$. Here, the red band represents uncertainty coming from the higher-loop contribution. We will see below that the radiative correction is comparable or even dominates the contribution of the higherdimensional operators $\left.\Delta m_{+}\right|_{\text {tree }}$ in a wide range of parameter region.

After all, the mass difference between the neutral and charged components is given by

$$
\Delta m_{+} \equiv m_{\widetilde{\chi}^{ \pm}}-m_{\widetilde{\chi}^{0}}=\left.\Delta m_{+}\right|_{\text {tree }}+\left.\Delta m_{+}\right|_{\text {rad }}
$$

where we define $m_{\widetilde{\chi}^{0}} \equiv m_{1}$. It plays an important role when we study the collider phenomenology of Higgsinos, as discussed in section 6.

In the following analysis, we use the above resummed dimension five operators for estimations of low-energy observables. As for contributions which cannot be covered only with the dimension five operators, we use the tree level result.

\footnotetext{
${ }^{4}$ We also give an analytic expression of $f(x)$ :

$$
f(x)=-x^{2}+x^{4} \ln (x)+4 x\left(1+\frac{x^{2}}{2}\right) \sqrt{1-\frac{x^{2}}{4}} \tan ^{-1}\left(\frac{2}{x} \sqrt{1-\frac{x^{2}}{4}}\right) .
$$
}




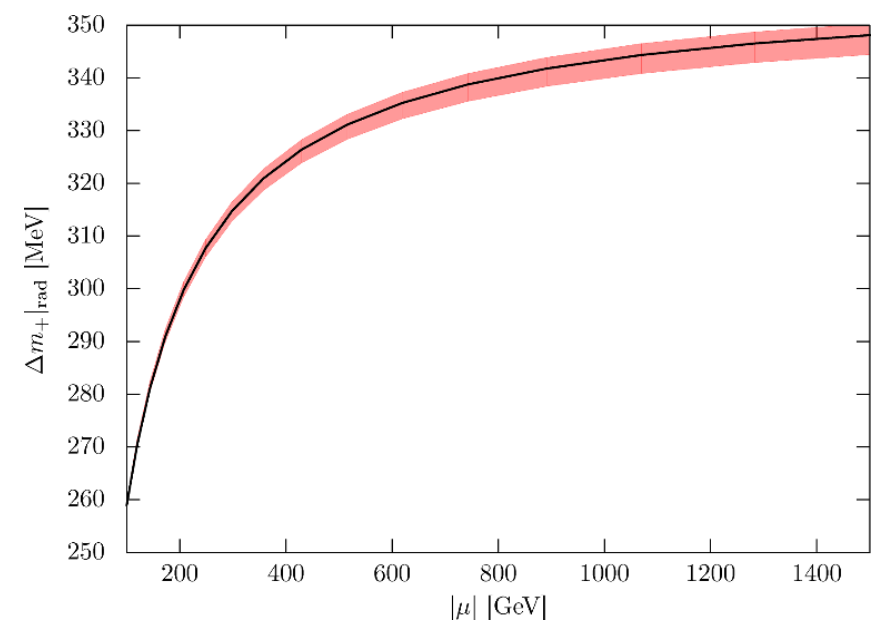

Figure 1. The radiative corrections to the neutral-charged Higgsino mass difference $\left.\Delta m_{+}\right|_{\mathrm{rad}}$ as a function of the Higgsino mass parameter $|\mu|$. Red band represents uncertainty coming from the higher-loop contribution.

\section{Renormalization of higher dimensional operators}

The dimension-five effective operators discussed above are induced by the Bino and Wino exchanging processes at the gaugino mass scale. Let us evaluate the matching conditions. First, we present our convention for the definition of the gaugino masses and the gauginoHiggsino-Higgs couplings. The gaugino mass terms are defined by

$$
\mathcal{L}_{\text {gaugino mass }}=-\frac{M_{1}}{2} \widetilde{B} \widetilde{B}-\frac{M_{2}}{2} \widetilde{W^{a}} \widetilde{W}^{a}+\text { h.c. }
$$

where $\widetilde{B}$ and $\widetilde{W}^{a}$ represent Bino and Wino, respectively, with $a$ being the $\mathrm{SU}(2)_{L}$ adjoint index. Relevant Yukawa interactions of the Higgs boson, Higgsinos and gauginos are given by

$$
\begin{aligned}
\mathcal{L}_{\mathrm{int}}= & -\frac{1}{\sqrt{2}}\left\{g_{1 u} H^{\dagger} \widetilde{H}_{u}+g_{1 d} \epsilon^{\alpha \beta}(H)_{\alpha}\left(\widetilde{H}_{d}\right)_{\beta}\right\} \widetilde{B} \\
& -\frac{1}{\sqrt{2}}\left\{g_{2 u} H^{\dagger} \sigma^{a} \tilde{H}_{u}-g_{2 d} \epsilon^{\alpha \beta}(H)_{\alpha}\left(\sigma^{a} \widetilde{H}_{d}\right)_{\beta}\right\} \widetilde{W}^{a}+\text { h.c. }
\end{aligned}
$$

where $\sigma^{a}$ are the Pauli matrices, and the above couplings at leading order are given as

$$
\begin{array}{ll}
g_{1 u}=g^{\prime} \sin \beta, & g_{1 d}=g^{\prime} \cos \beta, \\
g_{2 u}=g \sin \beta, & g_{2 d}=g \cos \beta,
\end{array}
$$

at the SUSY breaking scale. Here, $g^{\prime}$ is the $\mathrm{U}(1)_{Y}$ gauge coupling constant, and $\tan \beta \equiv$ $\left\langle H_{u}^{0}\right\rangle /\left\langle H_{d}^{0}\right\rangle$. Then, by integrating out the gauginos, we obtain the matching conditions for 


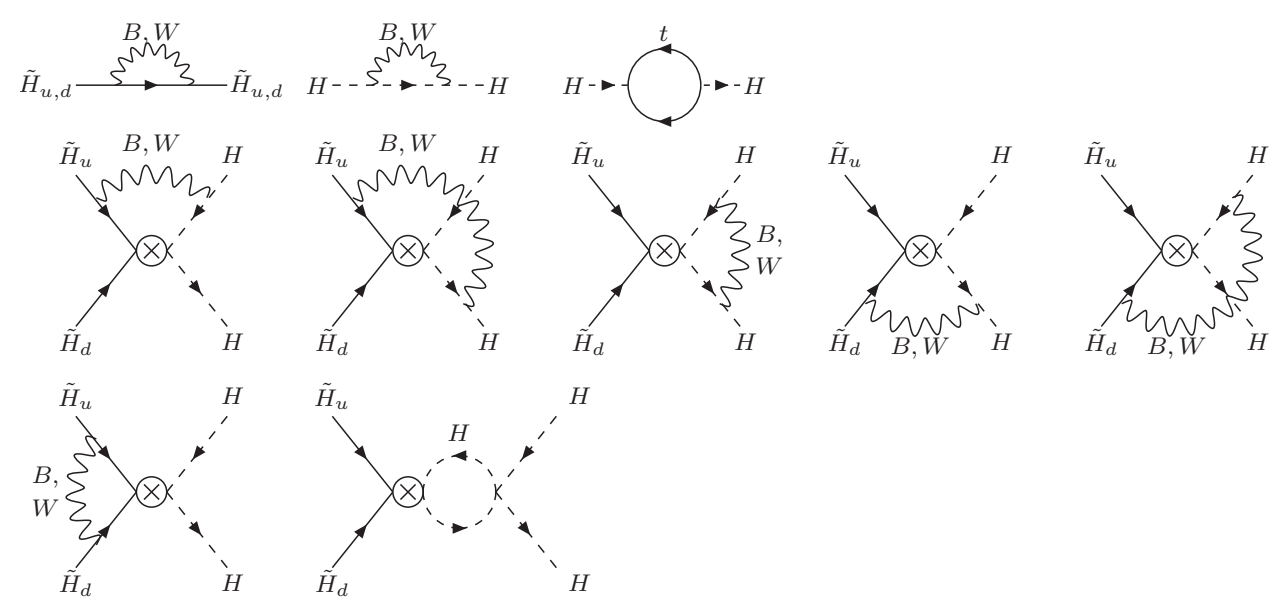

Figure 2. Examples of diagrams relevant for the RGEs.

the Wilson coefficients at the gaugino mass scale as follows:

$$
\begin{aligned}
c_{1} & =\frac{g_{1 u}^{2}}{4 M_{1}}+\frac{g_{2 u}^{2}}{4 M_{2}}, \\
c_{2} & =\frac{g_{1 d}^{2}}{4 M_{1}}+\frac{g_{2 d}^{2}}{4 M_{2}}, \\
d_{1} & =\frac{g_{1 u} g_{1 d}}{2 M_{1}}+\frac{g_{2 u} g_{2 d}}{2 M_{2}}, \\
d_{2} & =-\frac{g_{2 u} g_{2 d}}{M_{2}},
\end{aligned}
$$

with all of the parameters determined at the gaugino mass scale.

These Wilson coefficients are evolved down to the Higgsino mass scale according to the RGEs which we obtain by computing the diagrams in figure $2:^{5}$

$$
\frac{d c_{i}}{d \ln Q}=\frac{1}{16 \pi^{2}}\left(6 y_{t}^{2}+2 \lambda-3 g^{2}\right) c_{i},
$$

for $i=1,2$, and

$$
\frac{d}{d \ln Q}\left(d_{1}, d_{2}\right)=\left(d_{1}, d_{2}\right) \cdot \frac{1}{16 \pi^{2}}\left(\begin{array}{cc}
6 y_{t}^{2}+4 \lambda-3 g^{\prime 2}-6 g^{2} & -2 \lambda+3 g^{2} \\
-2 \lambda+3 g^{2} & 6 y_{t}^{2}+4 \lambda-3 g^{\prime 2}-6 g^{2}
\end{array}\right) .
$$

Here, $y_{t}$ is the top Yukawa coupling and $\lambda$ is the Higgs self-coupling given by

$$
\mathcal{L}_{\text {self }}=-\frac{\lambda}{2}\left(|H|^{2}\right)^{2},
$$

and we neglect the other Yukawa couplings than that of top quark.

To see the significance of the renormalization effects, as an example, we consider the case where the higher dimensional operators dominantly arise from the Wino exchange. At

${ }^{5}$ The RGE (3.5) can be read from that for the dimension-five operator for the neutrino masses [68, 69]. The RGEs for other coupling constants are presented in appendix C. 


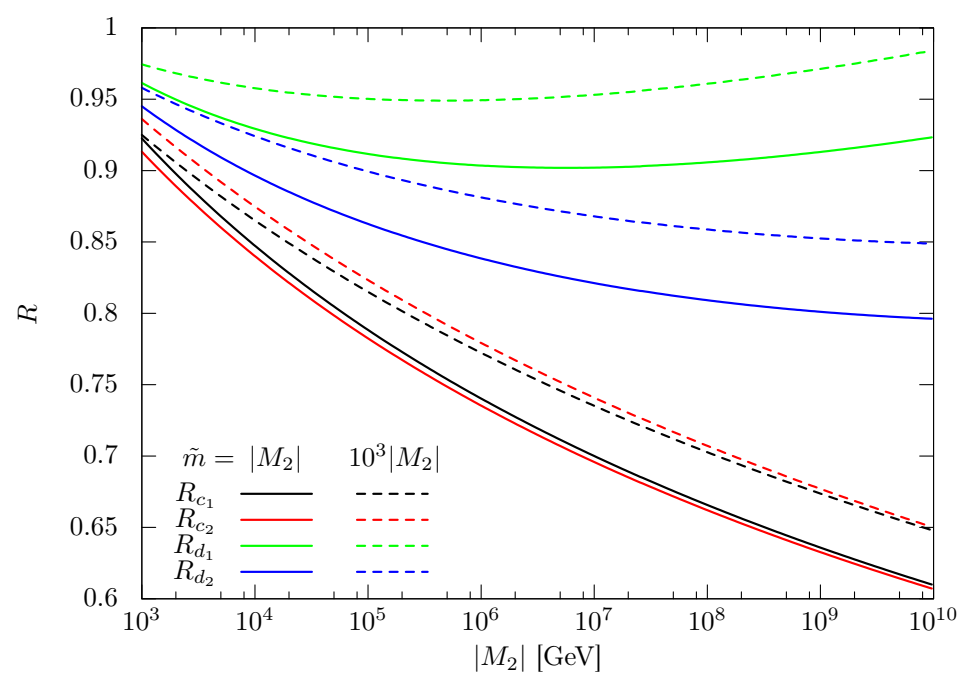

Figure 3. The ratios R's as functions the Wino mass. We set $\tan \beta=2$, and $\mu=100 \mathrm{GeV}$. Black, red, green, blue lines correspond to $R_{c_{1}}, R_{c_{2}}, R_{d_{1}}$, and $R_{d_{2}}$, respectively. Solid lines are for $\widetilde{m}=\left|M_{2}\right|$, while dashed lines for $\widetilde{m}=10^{3}\left|M_{2}\right|$.

tree level, we have

$$
\begin{array}{ll}
\left.c_{1} v^{2}\right|_{\text {tree }}=\frac{m_{W}^{2} \sin ^{2} \beta}{M_{2}}, & \left.c_{2} v^{2}\right|_{\text {tree }}=\frac{m_{W}^{2} \cos ^{2} \beta}{M_{2}}, \\
\left.d_{1} v^{2}\right|_{\text {tree }}=\frac{2 m_{W}^{2} \sin \beta \cos \beta}{M_{2}}, & \left.d_{2} v^{2}\right|_{\text {tree }}=\frac{-4 m_{W}^{2} \sin \beta \cos \beta}{M_{2}},
\end{array}
$$

with $m_{W}$ the $W$-boson mass. Let us define the ratio of the renormalized values to the tree level values, $R_{c_{i}}$ and $R_{d_{i}}(i=1,2)$ such that

$$
R_{c_{i}} \equiv \frac{c_{i}(|\mu|) v^{2}(|\mu|)}{\left.c_{i} v^{2}\right|_{\text {tree }}}, \quad R_{d_{i}} \equiv \frac{d_{i}(|\mu|) v^{2}(|\mu|)}{\left.d_{i} v^{2}\right|_{\text {tree }}} .
$$

Here we evaluate the running Higgs VEV $v$ according to ref. [70] as

$$
v^{2}(Q)=\frac{4\left\{m_{Z}^{2}+\operatorname{Re}\left[\Pi_{Z Z}^{T}\left(m_{Z}^{2}\right)\right]\right\}}{g^{\prime 2}(Q)+g^{2}(Q)},
$$

where $\Pi_{Z Z}^{T}\left(m_{Z}^{2}\right)$ is the transverse part of the $Z$-boson self-energy in the $\overline{\mathrm{MS}}$ scheme with external momentum set to be $p^{2}=m_{Z}^{2}$, and evaluated at the renormalization scale $Q$.

In figure 3 , we show the ratios $R_{c_{i}}$ and $R_{d_{i}}(i=1,2)$ as functions of the Wino mass $\left|M_{2}\right|$. Here we assume $\tan \beta=2$ and $\mu=100 \mathrm{GeV}$. The black, red, green, blue lines correspond to $R_{c_{1}}, R_{c_{2}}, R_{d_{1}}$, and $R_{d_{2}}$, respectively. In solid lines, we take the SUSY breaking scale $\widetilde{m}$ to be $\widetilde{m}=\left|M_{2}\right|$, while in dashed lines $\widetilde{m}=10^{3}\left|M_{2}\right|$. From this figure, we find that the renormalization group effects modify the Wilson coefficients by $\mathcal{O}(10) \%$. The difference is particularly important when one considers the mass difference in the Higgsino components, as we will see below. Moreover, the figure shows that the results depend not only on the Higgsino and gaugino masses, but also on the SUSY breaking scale $\widetilde{m}$. This is because 


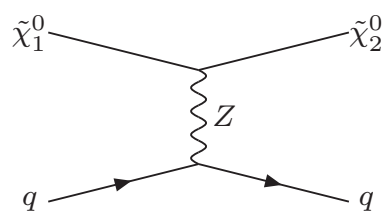

Figure 4. Diagram which gives rise to the inelastic scattering process $\widetilde{\chi}_{1}^{0} N \rightarrow \widetilde{\chi}_{2}^{0} N$.

the Higgsino-gaugino Yukawa couplings run differently from the gauge couplings below the SUSY breaking scale [5-7], ${ }^{6}$ and accordingly the relations (3.3) do not hold at the gaugino mass scale. This then affects the ratios $R_{c_{i}}$ and $R_{d_{i}}$, especially when the SUSY breaking scale is much higher than the gaugino mass scale.

\section{Higgsino dark matter search}

As mentioned in the Introduction, the neutral Higgsino LSP with a mass of around TeV scale can be a dark matter candidate. In fact, the thermal relic abundance of the Higgsino LSP is consistent with the observed DM density when it has $\sim 1 \mathrm{TeV}$ mass [61]. In this section, we assume that the Higgsino LSP occupies the dominant component of the DM in the Universe, and consider the constraints on the DM from the direct detection experiments. ${ }^{7}$ The mass of the Higgsino DM is assumed to be lower than $1 \mathrm{TeV}$ to satisfy the environment selection requirement discussed in the Introduction.

\subsection{Inelastic scattering}

Without the dimension-five effective operators, the Higgsino DM forms a Dirac fermion. In this case, the $Z$-boson exchange process induces the vector-vector coupling between the DM and a nucleon. Due to the coupling, the spin-independent (SI) scattering cross sections between the DM and nucleons are so large that this Dirac Higgsino scenario turns out to be already excluded by the direct detection experiments. However, thanks to the higher dimensional operators, the neutral components of Higgsino split into two Majorana fermions $\widetilde{\chi}_{1}^{0}$ and $\widetilde{\chi}_{2}^{0}$ with the mass difference $\Delta m$ given in eq. (2.14). Since a Majorana fermion does not have vector interactions, the Majorana Higgsino DM can avoid the bound from the direct detection experiments.

Nevertheless, if the mass difference $\Delta m$ is as small as $\mathcal{O}(100) \mathrm{keV}$, inelastic scattering processes $\widetilde{\chi}_{1}^{0} N \rightarrow \widetilde{\chi}_{2}^{0} N$ ( $N$ denotes a nucleon) may occur through the diagram in figure 4 . The inelastic scattering is also restricted by the direct detection experiments, depending on the mass difference $[72,73]$. Let us consider the constraints on the mass difference $\Delta m$ by studying the process. This bound then can be interpreted as an upper bound on the gaugino mass scale, as we will see in what follows.

\footnotetext{
${ }^{6}$ The RGEs of the Higgsino-gaugino couplings are given in appendix C. In addition, we have included finite threshold corrections at the SUSY breaking scale.

${ }^{7}$ As for the indirect search of the Higgsino DM, a robust limit is given in ref. [71] based on the observations of Milky Way's dwarf galaxies by Fermi Gamma-ray Space Telescope. According to the results, the current bound on the DM mass is $m_{\mathrm{DM}} \gtrsim 200-300 \mathrm{GeV}$.
} 
By evaluating the diagram in figure 4, we readily obtain the effective Lagrangian for the vector-vector interaction between the DM and quarks:

$$
\mathcal{L}_{\text {eff }}=b_{q} \overline{\widetilde{\chi}_{2}^{0}} \gamma^{\mu} \widetilde{\chi}_{1}^{0} \bar{q} \gamma_{\mu} q+\text { h.c. }
$$

with

$$
b_{q}=-\frac{i G_{F}}{\sqrt{2}}\left(T_{3}^{q}-2 Q_{q} \sin ^{2} \theta_{W}\right)
$$

where $G_{F}$ is the Fermi constant, and $T_{3}^{q}$ and $Q_{q}$ are $+1 / 2$ and $+2 / 3(-1 / 2$ and $-1 / 3)$ for up-type (down-type) quarks, respectively. Since sea quarks and gluons cannot contribute to the vector current, the effective vector couplings for proton and neutron are readily obtained as the sum of the valence quark contributions. By using the effective couplings, we obtain the SI inelastic scattering cross section of the Higgsino DM with a nucleus as

$$
\sigma_{\text {inelastic }}=\frac{G_{F}^{2}}{8 \pi}\left[N-\left(1-4 \sin ^{2} \theta_{W}\right) Z\right]^{2} M_{\text {red }}^{2},
$$

where $M_{\text {red }} \equiv m_{\widetilde{\chi}^{0}} m_{T} /\left(m_{\widetilde{\chi}^{0}}+m_{T}\right)$ is the reduced mass in the DM-nucleus system with $m_{T}$ being the mass of the target nucleus, and $Z$ and $N$ are the numbers of protons and neutrons in the nucleus, respectively. In the case of the ${ }^{131} \mathrm{Xe}$ target, for example, $Z=54$ and $N=77$ with a mass of $m_{T} \sim 122 \mathrm{GeV}$.

In a direct detection experiment, we search for the recoil energy $E_{R}$ of a target nucleus scattered off by the DM particle. The differential scattering rate for the Higgsino DM is expressed as

$$
\frac{d R}{d E_{R}}=\frac{N_{T} m_{T} \rho_{\widetilde{\chi}_{1}^{0}} G_{F}^{2}}{16 \pi m_{\widetilde{\chi}^{0}}}\left[N-\left(1-4 \sin ^{2} \theta_{W}\right) Z\right]^{2} F^{2}\left(E_{R}\right) \int_{v_{\min }}^{\infty} \frac{f(v)}{v} d v .
$$

Here, $N_{T}$ is the number of the target nuclei, $F^{2}\left(E_{R}\right)$ is a nuclear form factor, $\rho_{\widetilde{\chi}_{1}^{0}}$ is the local DM density, and $f(v)$ is the local DM velocity distribution. We use the same nuclear form factor as that given in ref. [74] in the following calculation. The DM density is assumed to be $\rho_{\widetilde{\chi}_{1}^{0}}=0.3 \mathrm{GeV} / \mathrm{cm}^{3}$. For $f(v)$, we use a Maxwell-Boltzmann velocity distribution with the escape velocity $v_{\text {esc }}$, in which the circular speed of the Sun is assumed to be $v_{0}=220 \mathrm{~km} / \mathrm{s}$. For the choice of the astrophysical parameters and the effects of their uncertainties on resultant constraints, see ref. [75]. In eq. (4.4), the minimum speed $v_{\text {min }}$ is given by

$$
v_{\min }=\frac{c}{\sqrt{2 m_{T} E_{R}}}\left(\frac{m_{T} E_{R}}{M_{\mathrm{red}}}+\Delta m\right)
$$

Dark matter direct detection experiments have good sensitivities for the recoil energy $E_{R}$ smaller than $\mathcal{O}(100) \mathrm{keV}$. Thus, if the mass difference $\Delta m$ is also smaller than $\mathcal{O}(100) \mathrm{keV}$, it significantly affects the direct detection rate. The effects enable us to probe or constraint $\Delta m$ in the region.

In figure 5 , we show the $90 \%$ C.L. lower limits on $\Delta m$ as functions of the DM mass $m_{\mathrm{DM}}$. The red, blue, and green bands show the constraints obtained from the data sets of the XENON10 $\left(E_{R}<250 \mathrm{keV}\right)$ [74], XENON100 $\left(E_{R}<50 \mathrm{keV}\right)$ [76], and LUX $\left(E_{R}<\right.$ $36 \mathrm{keV}$ ) [77] experiments, respectively. The upper (lower) line on each band corresponds 


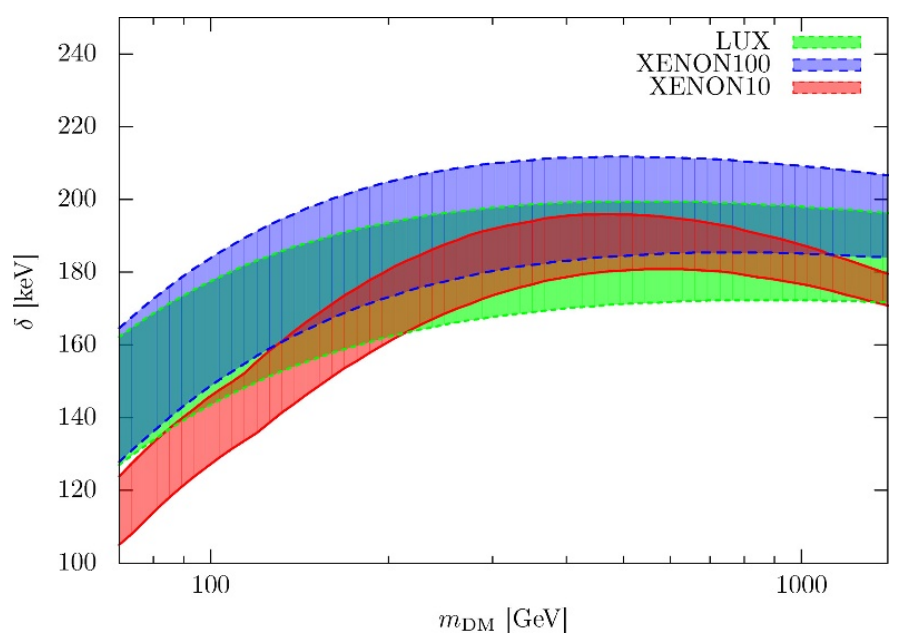

Figure 5. Lower limits on $\Delta m$ at $90 \%$ C.L as functions of DM mass $m_{\mathrm{DM}}$. Red, blue, and green bands are computed based on the data provided by the XENON10 [74], XENON100 [76], and LUX [77] experiments, respectively. Upper (lower) line on each band corresponds to $v_{\mathrm{esc}}=650$ $(500) \mathrm{km} / \mathrm{s}$.

to $v_{\text {esc }}=650(500) \mathrm{km} / \mathrm{s}$. To evaluate the limits, we have used the $p_{\max }$ method following ref. [78]. Slightly weaker limits are also provided in the XENON10 [74], CDMS II [79], and XENON100 [80] collaborations, though their analyses are optimized to the parameter regions which may account for the modulation observed by the DAMA/LIBRA experiment $[81,82]$. We find that, although the constraints highly depend on the astrophysical parameters such as the escape velocity $v_{\text {esc }}$, the current direct detection experiments have sensitivities to $\Delta m \lesssim(120-200) \mathrm{keV}$ in the case of the Higgsino DM scenario.

Now we interpret the above constraints in terms of the bounds on the gaugino mass scale. In the upper graph in figure 6 , we plot the mass differences $\Delta m$ as functions of the Wino mass $\left|M_{2}\right|$. Here, we take $\tan \beta=2, \mu=+500 \mathrm{GeV}, M_{1}=M_{2}$, and $\widetilde{m}=\left|M_{2}\right|$. The red-solid and green-dashed lines show the $\phi_{2} \equiv \arg \left(M_{2}\right)=0$ and $\pi / 2$ cases, respectively. Results for other phases lie between them. The dark (light) shaded region illustrates the weakest (strongest) limits depicted in figure 5. The limits show that $M_{2} \gtrsim 4 \times 10^{4} \mathrm{TeV}$ has been already excluded. Further, to see the size of the renormalization effects, we show in the lower graph the ratio of the mass differences computed with and without the resummation. It is found that to accurately extract the information on the gaugino mass scale, as well as the CP-nature in the gaugino-Higgsino system, to consider the renormalization effects is inevitable.

Before concluding this subsection, let us comment on the prospects of the Higgsino DM search based on the inelastic scattering. Unlike the XENON10 experiment, the current analyses of the XENON100 and LUX experiments are not optimized for the inelastic scattering. If the energy range analyzed in the LUX experiment is extended to $E_{R}=250 \mathrm{keV}$ with keeping the signal acceptance rate comparable to the present one, $\Delta m \sim 250$ (300) $\mathrm{keV}$ can be constrained for $v_{\text {esc }}=500(650) \mathrm{km} / \mathrm{s}$ and $m_{\mathrm{DM}}=500 \mathrm{GeV}$. We highly encourage such an analysis. 


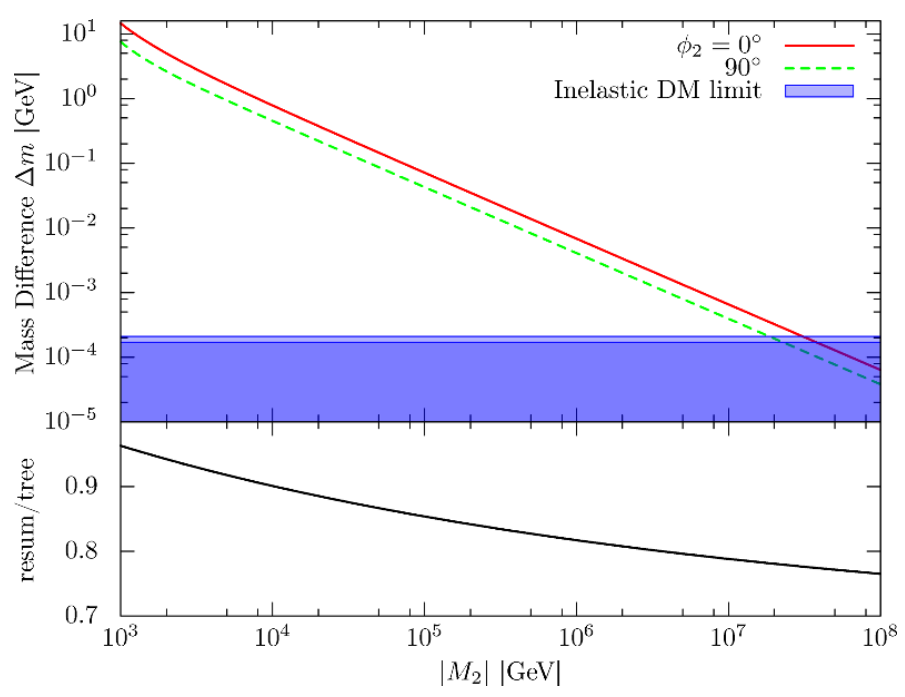

Figure 6. Mass difference $\Delta m$ as functions of the Wino mass $\left|M_{2}\right|$ in solid lines. Here, we take $\tan \beta=2, \mu=500 \mathrm{GeV}, M_{1}=M_{2}$, and $\widetilde{m}=\left|M_{2}\right|$. Red-solid and green-dashed lines show the $\phi_{2} \equiv \arg \left(M_{2}\right)=0$ and $\pi / 2$ cases, respectively. Dark (light) shaded region illustrates the weakest (strongest) bound given in figure 5 . The significance of the renormalization effects is shown in the lower graph.

\subsection{Elastic scattering}

In the presence of the higher-dimensional operators, the elastic scattering also occurs via the exchange of the Higgs boson and the $Z$-boson. The former gives rise to the SI scattering and the latter induces the spin-dependent (SD) one. In this subsection, we study these scattering processes. We will find that the SI scattering gives the lower bound on the gaugino mass scale, while the SD scattering is negligible.

The SI effective interactions between the DM and quarks/gluon are induced via the Higgs exchange processes. The SI effective couplings of the DM with quarks are generated by the diagram shown in figure 7 (a). They are expressed in terms of the effective operators as

$$
\mathcal{L}_{\text {eff }}=\sum_{q} f_{q} \overline{\widetilde{\chi}_{1}^{0}} \widetilde{\chi}_{1}^{0} m_{q} \bar{q} q
$$

with

$$
f_{q}=-\frac{1}{2 m_{h}^{2}} \operatorname{Re}\left[c_{1} e^{-i\left(\phi+\phi_{\mu}\right)}+c_{2} e^{i\left(\phi-\phi_{\mu}\right)}+d_{1} e^{-i \phi_{\mu}}\right] .
$$

Here, $m_{h}$ is the mass of the Higgs boson. From the expression, we find that the SI interactions depend on the CP phases in the Higgsino mass and the Wilson coefficients. With the coupling $f_{q}$, the Higgsino DM-nucleon effective coupling $f_{N}$ is written as

$$
\frac{f_{N}}{m_{N}}=\sum_{q=u, d, s} f_{q} f_{T_{q}}^{(N)}+\frac{2}{27} \sum_{Q=c, b, t} f_{Q} f_{T G}^{(N)},
$$

where $f_{T u}^{(p)}=0.019, f_{T d}^{(p)}=0.027, f_{T s}^{(p)}=0.009$ for proton and $f_{T u}^{(n)}=0.013, f_{T d}^{(n)}=0.040$, $f_{T s}^{(n)}=0.009$ for neutron, and $f_{T G}^{(N)} \equiv 1-\sum_{q=u, d, s} f_{T_{q}}^{(N)}$. They are computed from the recent 


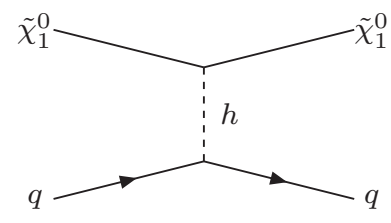

(a) SI

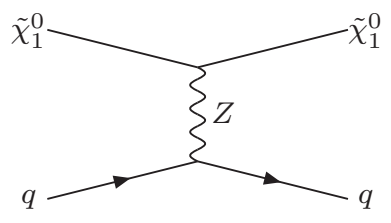

(b) SD

Figure 7. Diagrams induce the couplings of the Higgsino DM with quarks in the presence of the higher-dimensional operators.

results of the lattice QCD simulations [83, 84]. The SI elastic scattering cross section of the Higgsino DM with a target nucleus is then given as follows:

$$
\sigma_{\mathrm{SI}}=\frac{4}{\pi} M_{\mathrm{red}}^{2}\left(Z f_{p}+N f_{n}\right)^{2}
$$

In addition to the contribution, there exists the electroweak gauge boson contribution at loop-level. The contribution is presented in refs. [85, 86], and we take it into account in the following analysis.

The SD scattering is, on the other hand, induced by the $Z$-boson exchange process illustrated in figure $7(\mathrm{~b})$. The interactions are expressed in terms of the following effective Lagrangian:

$$
\mathcal{L}_{\mathrm{eff}}=d_{q} \overline{\widetilde{\chi}}_{1}^{0} \gamma^{\mu} \gamma_{5} \widetilde{\chi}_{1}^{0} \bar{q} \gamma_{\mu} \gamma_{5} q
$$

By evaluating the diagram, we obtain

$$
d_{q}=\frac{G_{F}}{\sqrt{2}} \cos 2 \theta T_{3}^{q} .
$$

Since the coupling is suppressed by $\cos 2 \theta$, and since the current experimental limits on the SD scattering are much weaker than those on the SI one, we can safely neglect the contribution in our scenario.

Figure 8 shows the SI scattering cross sections of the Higgsino DM with a proton as functions of $\left|M_{2}\right|$ in solid lines. Here we take $\tan \beta=2, \mu=500 \mathrm{GeV}, M_{1}=M_{2}$ and $\widetilde{m}=\left|M_{2}\right|$. The $\phi_{2}=\arg \left(M_{2}\right)=0, \pi / 2$ and $\pi$, cases are given in red-solid, green-dashed, and blue short-dashed lines, respectively, and another choice of the CP-phase falls between them. The upper blue-shaded region is already excluded by the LUX experiment [77]. The lower gray-shaded region represents the limitation of the direct detection experiments; once the experiments achieve the sensitivities to the cross sections they will suffer from the neutrino background and cannot distinguish the DM signal by means of the present technique [87]. In addition, we show the effects of the resummation on the calculation in the lower panel. As seen from the figure, the SI scattering cross sections highly depend on the CP-phase in the Higgsino-gaugino sector. When the gaugino scale is low enough, the future direct detection experiments may detect the signal of the DM. In higher gaugino mass regions, the electroweak loop effects dominate the contribution to the SI scattering cross sections and the resultant scattering cross sections become constant, though they are much lower than the neutrino background limit. 


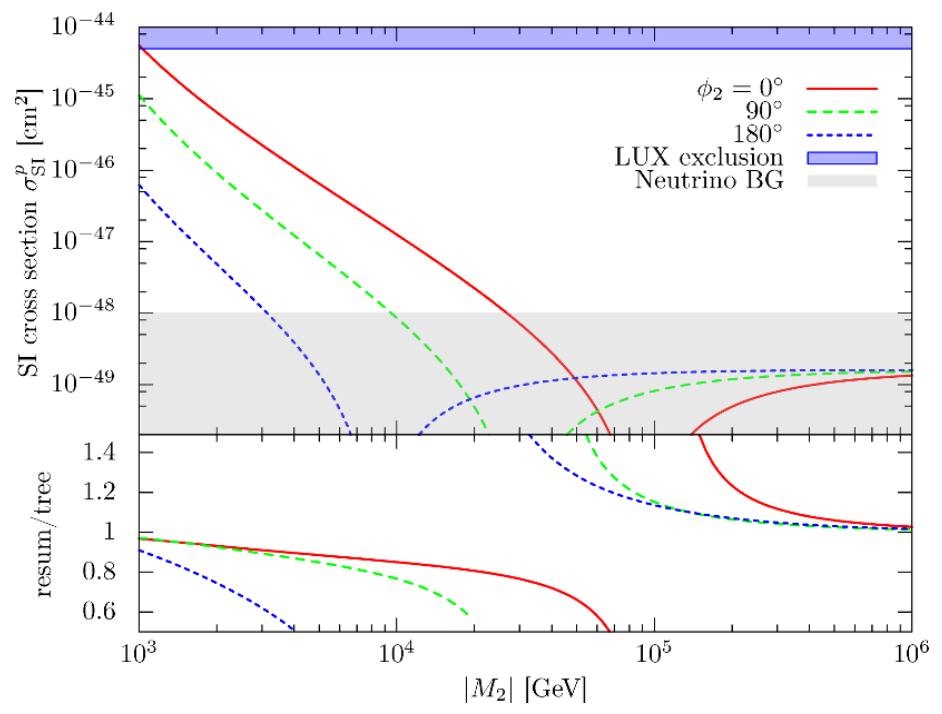

Figure 8. SI scattering cross sections of the Higgsino DM with a proton as functions of $\left|M_{2}\right|$ in solid lines. Here we take $\tan \beta=2, \mu=500 \mathrm{GeV}, M_{1}=M_{2}$ and $\widetilde{m}=\left|M_{2}\right|$. Red-solid, green-dashed, and blue short-dashed lines correspond to $\phi_{2}=\arg \left(M_{2}\right)=0, \pi / 2$ and $\pi$, respectively. Upper blueshaded region is excluded by the LUX experiment [77]. Lower gray-shaded region represents the limitation of the direct detection experiments due to the neutrino background [87]. Lower panel represents the effects of the resummation on the calculation.

\section{Electric dipole moments}

Generally, the MSSM induces new sources of CP violations, which may lead to large electric dipole moments (EDMs) of the SM fermions. One of the important contributions comes from one-loop diagrams which includes SUSY scalar particles. Another significant contribution is two-loop diagrams without the SUSY scalar particles. In the present "lonely Higgsino" scenario (typically when $\widetilde{m} \gg 10 \mathrm{TeV}$ ), the latter contribution is dominant.

As we noted above, the mass difference between the neutral components depends on the new CP phases in the effective interactions in eqs. (2.3) and (2.7), and their effects can be probed with the EDMs. The dominant contribution to the EDMs comes from the two-loop Barr-Zee diagrams [88] shown in figure 9 [89-91]. To evaluate the contribution, let us first show the Higgs-charged Higgsino vertex:

$$
\mathcal{L}_{\text {int }}=-\operatorname{Re}\left(d_{2}\right) v h \overline{\widetilde{H}^{+}} \widetilde{H}^{+}+\operatorname{Im}\left(d_{2}\right) v h \overline{\widetilde{H}^{+}} i \gamma_{5} \widetilde{H}^{+}
$$

and the $\mathrm{CP}$-odd part (the second term) is relevant to our calculation.

The definition of the EDMs of fermion $f$ is

$$
\mathcal{L}_{\mathrm{EDM}}=-\frac{i}{2} d_{f} \bar{f} \sigma^{\mu \nu} \gamma_{5} F_{\mu \nu} f
$$

We now evaluate the contribution of the diagrams in figure 9 to the EDM $d_{f}$. The result is given as follows [91]:

$$
d_{f}=d_{f}^{h \gamma}+d_{f}^{h Z}+d_{f}^{W W},
$$



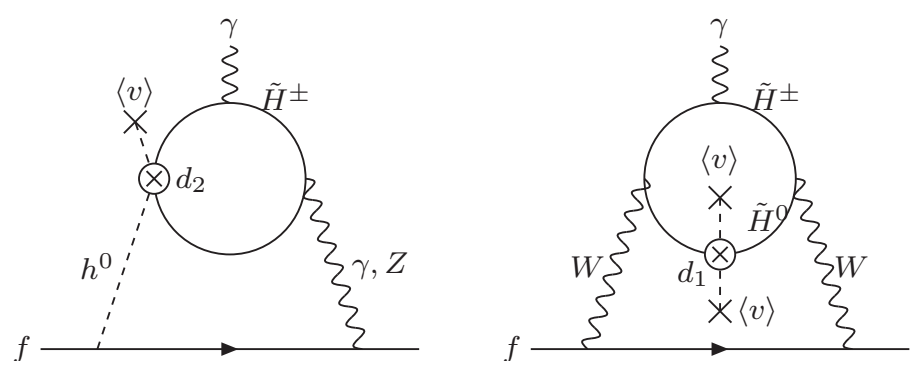

Figure 9. Two-loop Barr-Zee diagrams which give rise to the EDMs.

with

$$
\begin{aligned}
d_{f}^{h \gamma} & =\frac{4 e^{3} Q_{f} m_{f}}{(4 \pi)^{4}} \operatorname{Im}\left(\frac{d_{2}}{\mu}\right) f_{0}\left(\frac{|\mu|^{2}}{m_{h}^{2}}\right), \\
d_{f}^{h Z} & =\frac{e g^{2} m_{f}}{(4 \pi)^{4}}\left(T_{f}^{3}-2 Q_{f} \sin ^{2} \theta_{W}\right)\left(1-\tan ^{2} \theta_{W}\right) \operatorname{Im}\left(\frac{d_{2}}{\mu}\right) f_{1}\left(\frac{m_{Z}^{2}}{m_{h}^{2}}, \frac{|\mu|^{2}}{m_{h}^{2}}\right), \\
d_{f}^{W W} & =-\frac{e g^{2} m_{f} T_{f}^{3}}{(4 \pi)^{4}} \operatorname{Im}\left(\frac{d_{1}+d_{2}}{\mu}\right) f_{0}\left(\frac{|\mu|^{2}}{m_{W}^{2}}\right),
\end{aligned}
$$

where $Q_{f}, T_{f}$ and $m_{f}$ are the electric charge, isospin and mass of the fermion $f$, respectively, and $e$ is the electric charge of positron. The loop functions are given by ${ }^{8}$

$$
\begin{aligned}
f_{0}(r) & =r \int_{0}^{1} d x \frac{1}{r-x(1-x)} \ln \left(\frac{r}{x(1-x)}\right), \\
f_{1}\left(r_{1}, r_{2}\right) & =\frac{1}{1-r_{1}}\left[f_{0}\left(r_{2}\right)-r_{1} f_{0}\left(\frac{r_{2}}{r_{1}}\right)\right] .
\end{aligned}
$$

By using the expressions, we evaluate the electron EDM, which gives the most stringent bound on the Higgsino DM scenario at present. The results are given in figure 10. In the left graph, we plot each contribution to the electron EDM as a function of $\left|M_{2}\right|$. The red-solid, blue short-dashed, and green-dashed lines show the contribution of $-d_{e}^{h \gamma}, d_{e}^{W W}$, and $-d_{e}^{h Z}$, respectively. Here, we take $\tan \beta=2, \mu=500 \mathrm{GeV}, M_{1}=M_{2}, \phi_{2}=\pi / 2$, and $\widetilde{m}=\left|M_{2}\right|$. The blue-shaded region is excluded by the current experimental limit given by the ACME Collaboration [92]: $\left|d_{e}\right|<8.7 \times 10^{-29} \mathrm{ecm}$. The lower panel illustrates the renormalization effects. It is found that the $\gamma$ and $Z$-boson contributions have the opposite sign to the $W$-boson contribution. The suppression of the $Z$-boson contribution results from a numerically small factor of $T_{e}^{3}-2 Q_{e} \sin ^{2} \theta_{W}=-\left(1-4 \sin ^{2} \theta_{W}\right) / 2 \simeq-0.04$ in eq. (5.5). The total contribution is then shown in the right panel as a contour plot. Here, the red-solid and green-dashed lines represent the calculation with and without the resummation, respectively. As can be seen from the figure, the present experiments have

${ }^{8}$ Here, we also give the analytic expression of $f_{0}(r)$ for convenience:

$$
f_{0}(r)=\frac{2 r}{\sqrt{1-4 r}}\left[\ln (r) \ln \left(\frac{\sqrt{1-4 r}-1}{\sqrt{1-4 r}+1}\right)+\operatorname{Li}_{2}\left(\frac{2}{1-\sqrt{1-4 r}}\right)-\operatorname{Li}_{2}\left(\frac{2}{1+\sqrt{1-4 r}}\right)\right] .
$$




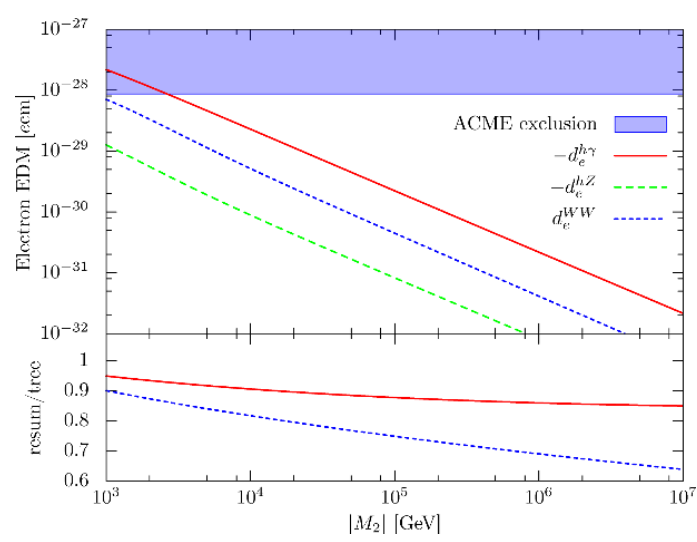

(a) Each contribution to the electron EDM.

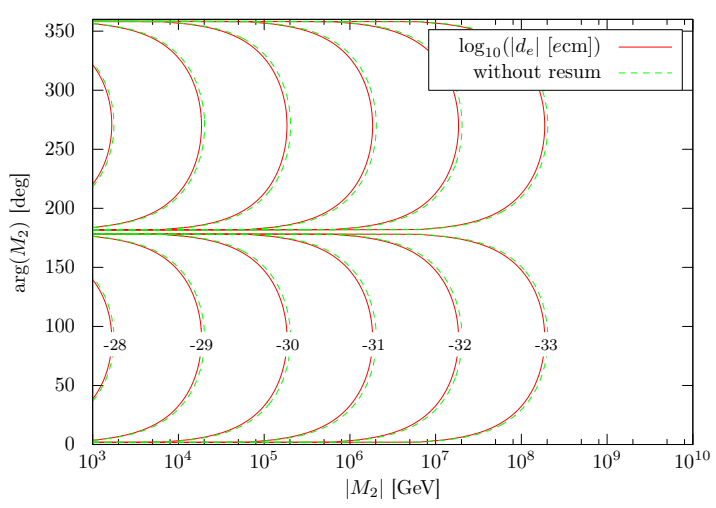

(b) Contour plot for the electron EDM.

Figure 10. Results for the electron EDM. We take $\tan \beta=2, \mu=500 \mathrm{GeV}, M_{1}=M_{2}$ and $\widetilde{m}=\left|M_{2}\right|$. Left: each contribution to the electron EDM as a function of $\left|M_{2}\right|$. Red-solid, blue short-dashed, and green-dashed lines show the contribution of $-d_{e}^{h \gamma}, d_{e}^{W W}$, and $-d_{e}^{h Z}$, respectively. We set $\phi_{2}=\pi / 2$. Blue-shaded region is excluded by the ACME experiment [92]. Lower panel illustrates the renormalization effects. Right: contour plot for the electron EDM. The red-solid and green-dashed lines represent the calculation with and without the resummation, respectively.

sensitivities to well above the $\mathrm{TeV}$ regime, and has already excluded a part of the parameter region shown in figure 10.

Future EDM experiments will have a few orders of magnitude improved sensitivity [93, 94], level of $d_{e} \sim 10^{-31} e \mathrm{~cm}$, or even smaller. In this case, the PeV scale gauginos can be probed.

\section{Collider signals}

As we have discussed above, the mass differences among the Higgsino-like chargino and neutralinos $\Delta m$ and $\Delta m_{+}$reflect the high-scale SUSY breaking parameters. Therefore, detailed measurements of the mass differences can reveal the high-energy physics. To that end, we also need to perform theoretical calculations for the mass differences accurately. The result for $\Delta m$ is already shown in figure 6 . In figure 11, we show a contour plot for the mass difference $\Delta m_{+}$in the $\arg \left(M_{2}\right)-\left|M_{2}\right|$ plane. Here, we take $\tan \beta=2$, $\mu=500 \mathrm{GeV}, M_{1}=M_{2}$, and $\widetilde{m}=\left|M_{2}\right|$. Red-solid and green-dashed lines show the calculations with and without the resummation effects, respectively. We find that when $\left|M_{2}\right|=\mathcal{O}(1) \mathrm{TeV}$ the chargino-neutralino mass difference can be as large as $\mathcal{O}(1) \mathrm{GeV}$. For heavier gaugino masses, on the other hand, the mass difference approaches to a constant value. This is because in this region the mass difference is determined by the electroweak loop contribution in eq. (2.24), and it reduces to $\left.\Delta m_{+}\right|_{\text {rad }} \simeq \alpha_{2} m_{Z} \sin ^{2} \theta_{W} / 2 \simeq 350 \mathrm{MeV}$ in the large gaugino mass limit as shown in figure 1.

In the case of $\Delta m_{+} \gtrsim m_{\pi}$, the chargino mainly decays into hadrons and a neutralino. The decay length of the chargino is [95]

$$
c \tau\left(\tilde{\chi}^{ \pm} \rightarrow \tilde{\chi}^{0} \pi^{ \pm}\right)=1.1 \mathrm{~cm}\left(\frac{\Delta m_{+}}{300 \mathrm{MeV}}\right)^{-3}\left[1-\frac{m_{\pi^{ \pm}}^{2}}{\Delta m_{+}^{2}}\right]^{-1 / 2} .
$$




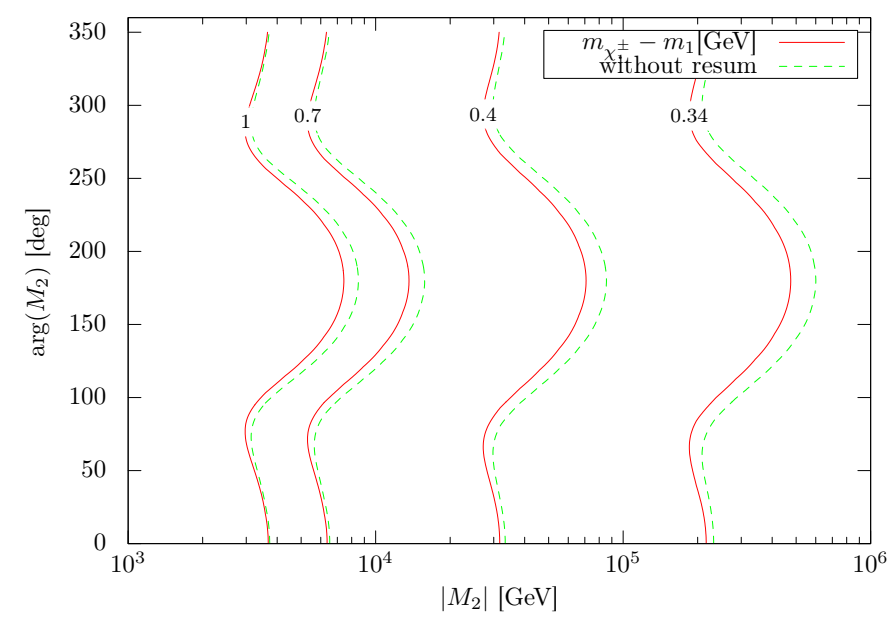

Figure 11. Contour plot for the mass difference $\Delta m_{+}$in the $\arg \left(M_{2}\right)-\left|M_{2}\right|$ plane. We take $\tan \beta=2, \mu=500 \mathrm{GeV}, M_{1}=M_{2}$, and $\widetilde{m}=\left|M_{2}\right|$. Red-solid and green-dashed lines show the calculations with and without the resummation effects, respectively.

In the case of the Higgsino LSP, $\Delta m_{+} \gtrsim 300 \mathrm{MeV}$, and thus it is difficult to directly detect a charged track of the chargino, unlike the Wino LSP case. In addition, smallness of the mass difference makes it hard to even discover the Higgsino at a hadron collider [96].

However, at lepton colliders, it is possible to identify SUSY particle production events by exploiting the hard photon tagging [97]. With the process $e^{+} e^{-} \rightarrow \tilde{\chi}^{+} \tilde{\chi}^{-} \gamma$, the LEP gives the lower limit on the chargino mass as $m_{\chi^{ \pm}} \gtrsim 90 \mathrm{GeV}$ [98]. At a future lepton collider, the measurement of the mass difference $\Delta m_{+}$to an accuracy of $O(1-10) \%$ is possible by observing the energy of the soft pion from the $\chi^{ \pm}$decay for $\Delta m_{+}=O(100) \mathrm{MeV}-$ $O(1) \mathrm{GeV}$ [99-101]. In this case, $\left.\Delta m_{+}\right|_{\text {tree }}>O(10) \mathrm{MeV}$ can be discriminated. In other words, a few tens of $\mathrm{TeV}$ gauginos can be probed by precisely measuring the chargino mass, as one can tell from figure 11. In the analysis performed in figure 12 in the subsequent section, we assume that a future lepton collider can determine the mass difference of the chargino with an accuracy of $20 \%$ and show the corresponding gaugino mass scale that can be probed with the mass measurements.

\section{Summary and discussion}

Finally, we summarize the results which have been obtained so far, and discuss the present constraints and future prospects on the Higgsino DM scenario. The plots in figure 12 show the result. Here, we set $\mu=+500 \mathrm{GeV}, M_{1}=M_{2}=M_{3}$ and $A$-terms are zero. The left plot shows the case of $\widetilde{m}=\left|M_{2}\right|$, while the right plot illustrates the $\widetilde{m}=10^{2}\left|M_{2}\right|$ case. The value of $\tan \beta$ is taken so that the Higgs mass is explained in the scenario. If an appropriate value of $\tan \beta \in[1: 50]$ is not found, it is set to be 1 (50) for the larger (smaller) Higgs mass. The mesh and shaded regions represent the present and future constraints, respectively. For the EDM, we include only the Barr-Zee contributions and omit the oneloop contribution with the sfermions in the plots. It turns out that the future experiments 


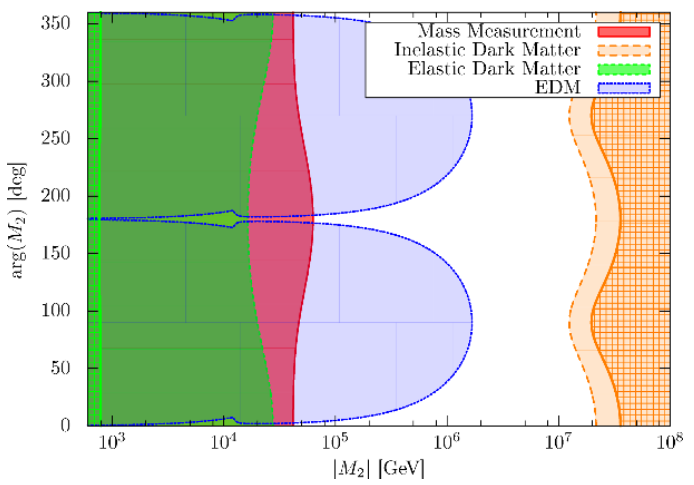

(a) $\widetilde{m}=\left|M_{2}\right|$

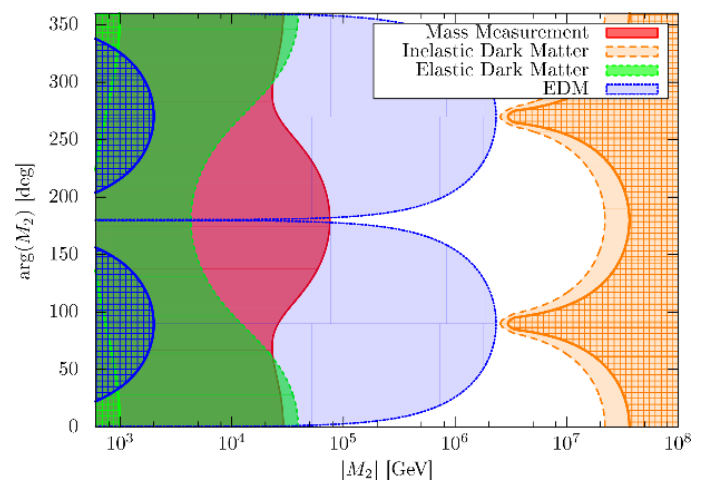

(b) $\tilde{m}=10^{2}\left|M_{2}\right|$

Figure 12. Current and future limits on the $\left|M_{2}\right|-\arg \left(M_{2}\right)$ space in the Higgsino DM scenario. Here, we set $\mu=+500 \mathrm{GeV}$ and $M_{1}=M_{2}=M_{3}$. As for the future sensitivity, we assume $\left|d_{e}\right|>10^{-31} e \mathrm{~cm}, \sigma_{\mathrm{SI}}>10^{-48} \mathrm{~cm}^{2}, \Delta m<300 \mathrm{keV}$ and $\left.\Delta m_{+}\right|_{\text {tree }}>\left.0.2 \Delta m_{+}\right|_{\mathrm{rad}}$.

have sensitivities to probe a wide range of parameter regions and are complementary to each other.

The heavier SUSY breaking scale can be also probed via measurement of the spectrum of the cosmic gravitational background [102]. This will give a good consistency check for the MSSM.

Although we exploit a bottom-up approach to discuss the Higgsino DM scenario in this paper, a top-down, or model-oriented approach is also possible. If we consider a concrete model in which the Higgsino LSP is realized, we may obtain some particular relations among the parameters in the model. Such a relation sometimes affects the nature of the Higgsino DM to a large extent. For example, let us consider a high-scale SUSY model discussed in ref. [27] where the Higgsino mass vanishes at tree level and is radiatively generated via the gaugino-Higgs loop diagrams. In this case, the relative phase between the Higgsino and gaugino mass terms is fixed: $\arg \left(\mu / M_{1}\right)=\arg \left(\mu / M_{2}\right)=\pi$. Thus, the EDMs are not generated in the scenario. Further, it turns out that the elastic scattering cross sections are also significantly suppressed. The reason is the following. The effective Higgsino-quark scalar coupling $f_{q}$ is given by

$$
f_{q} \simeq-\frac{g^{2}}{8 m_{h}^{2}}\left(\frac{\tan ^{2} \theta_{W}}{M_{1}}+\frac{1}{M_{2}}\right)(1-\sin 2 \beta)
$$

with the gaugino masses taken to be real and positive. On the other hand, to explain the mass of the Higgs boson in the scenario, $\tan \beta \simeq 1$ is favored. As a result, the effective coupling, and therefore the elastic scattering cross section as well, is extremely suppressed. The bound coming from the inelastic scattering is also evaded since the gaugino masses are $\mathcal{O}\left(10^{(2-3)}\right) \mathrm{TeV}$ to realize a viable Higgsino DM. Consequently, the experimental constraints on the scenario are significantly weakened.

In our work, we consider the effects of the SUSY particles on the Higgsino DM properties based on the effective theoretical formalism. The treatment is quite generic actually and 
applicable to other high-energy theories or DM models. A straightforward generalization of our study is to consider a generic multiplet of the $\mathrm{SU}(2)_{L} \otimes \mathrm{U}(1)_{L}$ gauge group with its neutral component assumed to be the DM - the so-called minimal DM scenario [61, 103, 104]. The effects of the high-energy physics on the DM are again described in terms of the higherdimensional operators, as discussed in refs. $[105,106]$. In this scenario, the viable region for the DM mass reaches as high as $\mathcal{O}(10) \mathrm{TeV}$. Thus, to thoroughly study the possibilities, the precision experiments discussed in this paper play a crucial role since it is much difficult to probe them in collider searches. This highly motivates subsequent works in this direction.

\section{Acknowledgments}

The work of N.N. is supported by Research Fellowships of the Japan Society for the Promotion of Science for Young Scientists.

\section{A Diagonalization of a $2 \times 2$ complex symmetric matrix}

Here we give a set of formulae for the diagonalization of a $2 \times 2$ complex symmetric matrix $M$ according to refs. $[107,108]$. Let us write the matrix as

$$
M=\left(\begin{array}{ll}
a & c \\
c & b
\end{array}\right)
$$

where $c \neq 0$ and $|a| \leq|b|$. We parametrize the $2 \times 2$ unitary matrix $U$ by

$$
U=\left(\begin{array}{cc}
e^{i \alpha} & 0 \\
0 & e^{i \beta}
\end{array}\right)\left(\begin{array}{cc}
\cos \theta & e^{-i \phi} \sin \theta \\
-e^{i \phi} \sin \theta & \cos \theta
\end{array}\right),
$$

which diagonalizes the matrix $M$ as

$$
U^{*} \mathcal{M} U^{\dagger}=\left(\begin{array}{cc}
m_{1} & 0 \\
0 & m_{2}
\end{array}\right)
$$

with $m_{1}$ and $m_{2}$ real and non-negative. Then, the above parameters are given as follows:

$$
\begin{aligned}
m_{1,2}^{2} & =\frac{1}{2}\left[|a|^{2}+|b|^{2}+2|c|^{2} \mp \sqrt{\left(|a|^{2}-|b|^{2}\right)^{2}+4\left|a^{*} c+b c^{*}\right|^{2}}\right] \\
\tan \theta & =\frac{|a|^{2}-|b|^{2}+\sqrt{\left(|a|^{2}-|b|^{2}\right)^{2}+4\left|a^{*} c+b c^{*}\right|^{2}}}{2\left|a^{*} c+b c^{*}\right|}, \\
e^{i \phi} & =\frac{a^{*} c+b c^{*}}{\left|a^{*} c+b c^{*}\right|}, \\
\alpha & =\frac{1}{2} \arg \left(a-c e^{-i \phi} \tan \theta\right), \\
\beta & =\frac{1}{2} \arg \left(b+c e^{i \phi} \tan \theta\right) .
\end{aligned}
$$




\section{B Higgsino gauge interactions in the mass eigenbasis}

In this section, we list the gauge interactions of Higgsinos in the mass eigenbasis, for convenience. Here, we use the four-component notation. The relevant interactions are given as follows:

$$
\mathcal{L}_{\text {gauge }}=\mathcal{L}_{W}+\mathcal{L}_{Z}+\mathcal{L}_{\gamma}
$$

with

$$
\begin{aligned}
& \mathcal{L}_{W}=-\frac{g e^{-\frac{i}{2} \phi}}{\sqrt{2}} \overline{\widetilde{\chi}^{+}} W^{+}\left[e^{\frac{i}{2}(\alpha+\gamma)} \sin \theta P_{L}+e^{-\frac{i}{2}(\alpha+\gamma)} \cos \theta P_{R}\right] \widetilde{\chi}_{1}^{0} \\
& -\frac{i g e^{-\frac{i}{2} \phi}}{\sqrt{2}} \overline{\widetilde{\chi}^{+}} W^{+}\left[e^{\frac{i}{2}(\beta+\gamma)} \sin \theta P_{L}+e^{-\frac{i}{2}(\beta+\gamma)} \cos \theta P_{R}\right] \widetilde{\chi}_{2}^{0}+\text { h.c. }, \\
& \mathcal{L}_{Z}=+\frac{g_{Z}}{2}\left(1-2 \sin ^{2} \theta_{W}\right) \overline{\widetilde{\chi}^{+}} \not{\psi} \widetilde{\chi}^{+} \\
& +\frac{i g_{Z}}{4}\left[\overline{\widetilde{\chi}_{2}^{0}} \boldsymbol{\psi} \widetilde{\chi}_{1}^{0}-\overline{\widetilde{\chi}_{1}^{0}} \boldsymbol{\psi} \widetilde{\chi}_{2}^{0}\right] \\
& +\frac{g_{Z}}{8}(\alpha-\beta)\left[\overline{\widetilde{\chi}_{2}^{0}} Z \gamma_{5} \widetilde{\chi}_{1}^{0}+\overline{\widetilde{\chi}_{1}^{0}} Z \gamma_{5} \widetilde{\chi}_{2}^{0}\right] \\
& -\frac{g_{Z}}{4} \cos 2 \theta\left[\overline{\tilde{\chi}_{1}^{0}} Z \gamma_{5} \widetilde{\chi}_{1}^{0}-\overline{\widetilde{\chi}_{2}^{0}} Z \gamma_{5} \widetilde{\chi}_{2}^{0}\right] \text {, } \\
& \mathcal{L}_{\gamma}=-e \overline{\widetilde{\chi}^{+}} A \widetilde{\chi}^{+},
\end{aligned}
$$

where $P_{L / R} \equiv\left(1 \mp \gamma_{5}\right) / 2$ and $g_{Z} \equiv \sqrt{g^{2}+g^{\prime 2}}$.

\section{Renormalization group equations}

Here, we present the RGEs other than those in the SM which are used in the above calculation. First of all, the RGEs of the gauge couplings are written as

$$
\frac{d g_{A}}{d \ln Q}=\frac{b_{A} g_{A}^{3}}{16 \pi^{2}}
$$

where $g_{1}=g^{\prime}, g_{2}=g$, and $g_{3}=g_{s}$ is the strong gauge coupling constant. Above the Higgsino threshold, the one-loop beta-function coefficients $b_{A}$ are given by $\left(b_{1}, b_{2}, b_{3}\right)=$ $\left(\frac{15}{2},-\frac{5}{2},-7\right)$. After gauginos show up, we use $\left(b_{1}, b_{2}, b_{3}\right)=\left(\frac{15}{2},-\frac{7}{6},-5\right)$.

Below the SUSY breaking scale, the running of the gaugino couplings differs from that of the gauge couplings [5-7]. The RGEs of the gaugino couplings $g_{i u}$ and $g_{i d}(i=1,2)$ in eq. (3.2) are

$$
\begin{aligned}
\frac{d g_{1 u}}{d \ln Q} & =\frac{1}{16 \pi^{2}}\left[g_{1 u}\left(\frac{3}{4} g_{1 u}^{2}+\frac{3}{2} g_{1 d}^{2}+\frac{3}{4} g_{2 u}^{2}+3 y_{t}^{2}-\frac{3}{4} g^{\prime 2}-\frac{9}{4} g^{2}\right)+3 g_{1 d} g_{2 u} g_{2 d}\right], \\
\frac{d g_{1 d}}{d \ln Q} & =\frac{1}{16 \pi^{2}}\left[g_{1 d}\left(\frac{3}{4} g_{1 d}^{2}+\frac{3}{2} g_{1 u}^{2}+\frac{3}{4} g_{2 d}^{2}+3 y_{t}^{2}-\frac{3}{4} g^{\prime 2}-\frac{9}{4} g^{2}\right)+3 g_{1 u} g_{2 u} g_{2 d}\right], \\
\frac{d g_{2 u}}{d \ln Q} & =\frac{1}{16 \pi^{2}}\left[g_{2 u}\left(\frac{5}{4} g_{2 u}^{2}-\frac{1}{2} g_{2 d}^{2}+\frac{1}{4} g_{1 u}^{2}+3 y_{t}^{2}-\frac{3}{4} g^{\prime 2}-\frac{33}{4} g^{2}\right)+g_{2 d} g_{1 u} g_{1 d}\right], \\
\frac{d g_{2 d}}{d \ln Q} & =\frac{1}{16 \pi^{2}}\left[g_{2 d}\left(\frac{5}{4} g_{2 d}^{2}-\frac{1}{2} g_{2 u}^{2}+\frac{1}{4} g_{1 d}^{2}+3 y_{t}^{2}-\frac{3}{4} g^{\prime 2}-\frac{33}{4} g^{2}\right)+g_{2 u} g_{1 u} g_{1 d}\right],
\end{aligned}
$$


while that of the top Yukawa coupling at one-loop level is given by

$$
\frac{d y_{t}}{d \ln Q}=\frac{1}{16 \pi^{2}}\left[\frac{9}{2} y_{t}^{2}-\frac{17}{12} g^{\prime 2}-\frac{9}{4} g^{2}-8 g_{s}^{2}+\frac{1}{2}\left(g_{1 u}^{2}+g_{1 d}^{2}\right)+\frac{3}{2}\left(g_{2 u}^{2}+g_{2 d}^{2}\right)\right] y_{t} .
$$

Open Access. This article is distributed under the terms of the Creative Commons Attribution License (CC-BY 4.0), which permits any use, distribution and reproduction in any medium, provided the original author(s) and source are credited.

\section{References}

[1] ATLAS collaboration, Observation of a new particle in the search for the Standard Model Higgs boson with the ATLAS detector at the LHC, Phys. Lett. B 716 (2012) 1 [arXiv:1207.7214] [INSPIRE].

[2] CMS collaboration, Observation of a new boson at a mass of $125 \mathrm{GeV}$ with the CMS experiment at the LHC, Phys. Lett. B 716 (2012) 30 [arXiv:1207.7235] [INSPIRE].

[3] J.D. Wells, Implications of supersymmetry breaking with a little hierarchy between gauginos and scalars, hep-ph/0306127 [INSPIRE].

[4] J.D. Wells, PeV-scale supersymmetry, Phys. Rev. D 71 (2005) 015013 [hep-ph/0411041] [INSPIRE].

[5] N. Arkani-Hamed and S. Dimopoulos, Supersymmetric unification without low energy supersymmetry and signatures for fine-tuning at the LHC, JHEP 06 (2005) 073 [hep-th/0405159] [INSPIRE].

[6] G.F. Giudice and A. Romanino, Split supersymmetry, Nucl. Phys. B 699 (2004) 65 [Erratum ibid. B 706 (2005) 65] [hep-ph/0406088] [INSPIRE].

[7] N. Arkani-Hamed, S. Dimopoulos, G.F. Giudice and A. Romanino, Aspects of split supersymmetry, Nucl. Phys. B 709 (2005) 3 [hep-ph/0409232] [INSPIRE].

[8] N. Arkani-Hamed, S. Dimopoulos and S. Kachru, Predictive landscapes and new physics at a $\mathrm{TeV}$, hep-th/0501082 [INSPIRE].

[9] Y. Okada, M. Yamaguchi and T. Yanagida, Upper bound of the lightest Higgs boson mass in the minimal supersymmetric standard model, Prog. Theor. Phys. 85 (1991) 1 [INSPIRE].

[10] Y. Okada, M. Yamaguchi and T. Yanagida, Renormalization group analysis on the Higgs mass in the softly broken supersymmetric standard model, Phys. Lett. B 262 (1991) 54 [INSPIRE].

[11] J.R. Ellis, G. Ridolfi and F. Zwirner, Radiative corrections to the masses of supersymmetric Higgs bosons, Phys. Lett. B 257 (1991) 83 [INSPIRE].

[12] H.E. Haber and R. Hempfling, Can the mass of the lightest Higgs boson of the minimal supersymmetric model be larger than m(Z)?, Phys. Rev. Lett. 66 (1991) 1815 [INSPIRE].

[13] J.R. Ellis, G. Ridolfi and F. Zwirner, On radiative corrections to supersymmetric Higgs boson masses and their implications for LEP searches, Phys. Lett. B 262 (1991) 477 [INSPIRE].

[14] G.F. Giudice and A. Strumia, Probing High-Scale and Split Supersymmetry with Higgs Mass Measurements, Nucl. Phys. B 858 (2012) 63 [arXiv:1108.6077] [INSPIRE].

[15] J. Hisano, T. Kuwahara and N. Nagata, Grand Unification in High-scale Supersymmetry, Phys. Lett. B 723 (2013) 324 [arXiv:1304.0343] [INSPIRE]. 
[16] J. Hisano, D. Kobayashi, T. Kuwahara and N. Nagata, Decoupling Can Revive Minimal Supersymmetric SU(5), JHEP 07 (2013) 038 [arXiv: 1304.3651] [INSPIRE].

[17] N. Nagata and S. Shirai, Sfermion Flavor and Proton Decay in High-Scale Supersymmetry, JHEP 03 (2014) 049 [arXiv: 1312.7854] [INSPIRE].

[18] F. Gabbiani, E. Gabrielli, A. Masiero and L. Silvestrini, A Complete analysis of FCNC and $C P$ constraints in general SUSY extensions of the standard model, Nucl. Phys. B $\mathbf{4 7 7}$ (1996) 321 [hep-ph/9604387] [INSPIRE].

[19] T. Moroi and M. Nagai, Probing Supersymmetric Model with Heavy Sfermions Using Leptonic Flavor and CP-violations, Phys. Lett. B 723 (2013) 107 [arXiv:1303.0668] [INSPIRE].

[20] D. McKeen, M. Pospelov and A. Ritz, Electric dipole moment signatures of PeV-scale superpartners, Phys. Rev. D 87 (2013) 113002 [arXiv:1303.1172] [InSPIRE].

[21] R. Sato, S. Shirai and K. Tobioka, Flavor of Gluino Decay in High-Scale Supersymmetry, JHEP 10 (2013) 157 [arXiv: 1307.7144] [INSPIRE].

[22] W. Altmannshofer, R. Harnik and J. Zupan, Low Energy Probes of PeV Scale Sfermions, JHEP 11 (2013) 202 [arXiv:1308.3653] [INSPIRE].

[23] K. Fuyuto, J. Hisano, N. Nagata and K. Tsumura, QCD Corrections to Quark (Chromo)Electric Dipole Moments in High-scale Supersymmetry, JHEP 12 (2013) 010 [arXiv: 1308.6493] [INSPIRE].

[24] S. Weinberg, Cosmological Constraints on the Scale of Supersymmetry Breaking, Phys. Rev. Lett. 48 (1982) 1303 [INSPIRE].

[25] M. Kawasaki, K. Kohri and T. Moroi, Hadronic decay of the gravitino in the early universe and its implications to inflation, hep-ph/0410287 [INSPIRE].

[26] M. Kawasaki, K. Kohri, T. Moroi and A. Yotsuyanagi, Big-Bang Nucleosynthesis and Gravitino, Phys. Rev. D 78 (2008) 065011 [arXiv:0804.3745] [INSPIRE].

[27] L.J. Hall and Y. Nomura, Spread Supersymmetry, JHEP 01 (2012) 082 [arXiv:1111.4519] [INSPIRE].

[28] L.J. Hall, Y. Nomura and S. Shirai, Spread supersymmetry with Wino LSP: gluino and dark matter signals, JHEP 01 (2013) 036 [arXiv:1210.2395] [INSPIRE].

[29] M. Ibe and T.T. Yanagida, The Lightest Higgs Boson Mass in Pure Gravity Mediation Model, Phys. Lett. B 709 (2012) 374 [arXiv:1112.2462] [INSPIRE].

[30] M. Ibe, S. Matsumoto and T.T. Yanagida, Pure Gravity Mediation with $m_{3 / 2}=10-100 \mathrm{TeV}$, Phys. Rev. D 85 (2012) 095011 [arXiv:1202.2253] [INSPIRE].

[31] A. Arvanitaki, N. Craig, S. Dimopoulos and G. Villadoro, Mini-Split, JHEP 02 (2013) 126 [arXiv:1210.0555] [INSPIRE].

[32] N. Arkani-Hamed, A. Gupta, D.E. Kaplan, N. Weiner and T. Zorawski, Simply Unnatural Supersymmetry, arXiv:1212.6971 [INSPIRE].

[33] V. Agrawal, S.M. Barr, J.F. Donoghue and D. Seckel, The Anthropic principle and the mass scale of the standard model, Phys. Rev. D 57 (1998) 5480 [hep-ph/9707380] [INSPIRE].

[34] S. Weinberg, Anthropic Bound on the Cosmological Constant, Phys. Rev. Lett. 59 (1987) 2607 [INSPIRE]. 
[35] G. Elor, H.-S. Goh, L.J. Hall, P. Kumar and Y. Nomura, Environmentally Selected WIMP Dark Matter with High-Scale Supersymmetry Breaking, Phys. Rev. D 81 (2010) 095003 [arXiv: 0912.3942] [INSPIRE].

[36] L.J. Hall and Y. Nomura, Grand Unification and Intermediate Scale Supersymmetry, JHEP 02 (2014) 129 [arXiv:1312.6695] [INSPIRE].

[37] Y. Nomura and S. Shirai, Supersymmetry from Typicality: TeV-Scale Gauginos and PeV-Scale Squarks and Sleptons, Phys. Rev. Lett. 113 (2014) 111801 [arXiv:1407.3785] [INSPIRE].

[38] M. Tegmark, A. Aguirre, M. Rees and F. Wilczek, Dimensionless constants, cosmology and other dark matters, Phys. Rev. D 73 (2006) 023505 [astro-ph/0511774] [INSPIRE].

[39] L.J. Hall, Y. Nomura and S. Shirai, Grand Unification, Axion and Inflation in Intermediate Scale Supersymmetry, JHEP 06 (2014) 137 [arXiv:1403.8138] [INSPIRE].

[40] BICEP2 collaboration, P.A.R. Ade et al., Detection of B-Mode Polarization at Degree Angular Scales by BICEP2, Phys. Rev. Lett. 112 (2014) 241101 [arXiv:1403.3985] [INSPIRE].

[41] Planck collaboration, R. Adam et al., Planck intermediate results. XXX. The angular power spectrum of polarized dust emission at intermediate and high Galactic latitudes, arXiv: 1409.5738 [INSPIRE].

[42] M. Fukugita and T. Yanagida, Baryogenesis Without Grand Unification, Phys. Lett. B 174 (1986) 45 [inSPIRE].

[43] T. Gherghetta, G.F. Giudice and J.D. Wells, Phenomenological consequences of supersymmetry with anomaly induced masses, Nucl. Phys. B 559 (1999) 27 [hep-ph/9904378] [INSPIRE].

[44] T. Moroi and L. Randall, Wino cold dark matter from anomaly mediated SUSY breaking, Nucl. Phys. B 570 (2000) 455 [hep-ph/9906527] [INSPIRE].

[45] L. Randall and R. Sundrum, Out of this world supersymmetry breaking, Nucl. Phys. B 557 (1999) 79 [hep-th/9810155] [inSPIRE].

[46] G.F. Giudice, M.A. Luty, H. Murayama and R. Rattazzi, Gaugino mass without singlets, JHEP 12 (1998) 027 [hep-ph/9810442] [inSPIRE].

[47] J. Hisano, S. Matsumoto and M.M. Nojiri, Explosive dark matter annihilation, Phys. Rev. Lett. 92 (2004) 031303 [hep-ph/0307216] [INSPIRE].

[48] J. Hisano, S. Matsumoto, M.M. Nojiri and O. Saito, Non-perturbative effect on dark matter annihilation and gamma ray signature from galactic center, Phys. Rev. D 71 (2005) 063528 [hep-ph/0412403] [INSPIRE].

[49] J. Hisano, S. Matsumoto, M. Nagai, O. Saito and M. Senami, Non-perturbative effect on thermal relic abundance of dark matter, Phys. Lett. B 646 (2007) 34 [hep-ph/0610249] [INSPIRE].

[50] N. Arkani-Hamed, A. Delgado and G.F. Giudice, The Well-tempered neutralino, Nucl. Phys. B 741 (2006) 108 [hep-ph/0601041] [INSPIRE].

[51] S. Shirai, F. Takahashi and T.T. Yanagida, R-violating Decay of Wino Dark Matter and electron/positron Excesses in the PAMELA/Fermi Experiments, Phys. Lett. B 680 (2009) 485 [arXiv:0905.0388] [INSPIRE]. 
[52] M. Ibe, S. Matsumoto, S. Shirai and T.T. Yanagida, AMS-02 Positrons from Decaying Wino in the Pure Gravity Mediation Model, JHEP 07 (2013) 063 [arXiv:1305.0084] [INSPIRE].

[53] M. Ibe, S. Matsumoto, S. Shirai and T.T. Yanagida, Mass of Decaying Wino from AMS-02 2014, arXiv:1409.6920 [INSPIRE].

[54] J. Hisano, K. Ishiwata and N. Nagata, A complete calculation for direct detection of Wino dark matter, Phys. Lett. B 690 (2010) 311 [arXiv:1004.4090] [INSPIRE].

[55] J. Hisano, K. Ishiwata and N. Nagata, Gluon contribution to the dark matter direct detection, Phys. Rev. D 82 (2010) 115007 [arXiv:1007.2601] [INSPIRE].

[56] M. Ibe, S. Matsumoto and R. Sato, Mass Splitting between Charged and Neutral Winos at Two-Loop Level, Phys. Lett. B 721 (2013) 252 [arXiv:1212.5989] [INSPIRE].

[57] T. Cohen, M. Lisanti, A. Pierce and T.R. Slatyer, Wino Dark Matter Under Siege, JCAP 10 (2013) 061 [arXiv: 1307.4082] [INSPIRE].

[58] J. Fan and M. Reece, In Wino Veritas? Indirect Searches Shed Light on Neutralino Dark Matter, JHEP 10 (2013) 124 [arXiv:1307.4400] [INSPIRE].

[59] B. Bhattacherjee, M. Ibe, K. Ichikawa, S. Matsumoto and K. Nishiyama, Wino Dark Matter and Future dSph Observations, JHEP 07 (2014) 080 [arXiv:1405.4914] [INSPIRE].

[60] R.D. Peccei and H.R. Quinn, CP Conservation in the Presence of Instantons, Phys. Rev. Lett. 38 (1977) 1440 [INSPIRE].

[61] M. Cirelli, A. Strumia and M. Tamburini, Cosmology and Astrophysics of Minimal Dark Matter, Nucl. Phys. B 787 (2007) 152 [arXiv:0706.4071] [InSPIRE].

[62] K. Cheung, C.-W. Chiang and J. Song, A Minimal supersymmetric scenario with only mu at the weak scale, JHEP 04 (2006) 047 [hep-ph/0512192] [INSPIRE].

[63] K. Cheung, S.Y. Choi and J. Song, Impact on the Light Higgsino-LSP Scenario from Physics beyond the Minimal Supersymmetric Standard Model, Phys. Lett. B 677 (2009) 54 [arXiv: 0903.3175] [INSPIRE].

[64] V.A. Beylin, V.I. Kuksa, G.M. Vereshkov and R.S. Pasechnik, Neutralino-nucleon interaction in the Split Higgsino scenario of the Dark Matter, Int. J. Mod. Phys. A 24 (2009) 6051 [arXiv: 0903.4201] [INSPIRE].

[65] K.S. Jeong, M. Shimosuka and M. Yamaguchi, Light Higgsino in Heavy Gravitino Scenario with Successful Electroweak Symmetry Breaking, JHEP 09 (2012) 050 [arXiv:1112.5293] [INSPIRE].

[66] P.J. Fox, G.D. Kribs and A. Martin, Split Dirac Supersymmetry: An Ultraviolet Completion of Higgsino Dark Matter, Phys. Rev. D 90 (2014) 075006 [arXiv:1405.3692] [INSPIRE].

[67] R. Essig, Direct Detection of Non-Chiral Dark Matter, Phys. Rev. D 78 (2008) 015004 [arXiv:0710.1668] [INSPIRE].

[68] P.H. Chankowski and Z. Pluciennik, Renormalization group equations for seesaw neutrino masses, Phys. Lett. B 316 (1993) 312 [hep-ph/9306333] [INSPIRE].

[69] K.S. Babu, C.N. Leung and J.T. Pantaleone, Renormalization of the neutrino mass operator, Phys. Lett. B 319 (1993) 191 [hep-ph/9309223] [INSPIRE].

[70] D.M. Pierce, J.A. Bagger, K.T. Matchev and R.-j. Zhang, Precision corrections in the minimal supersymmetric standard model, Nucl. Phys. B 491 (1997) 3 [hep-ph/9606211] [INSPIRE]. 
[71] A. Geringer-Sameth, S.M. Koushiappas and M.G. Walker, A Comprehensive Search for Dark Matter Annihilation in Dwarf Galaxies, arXiv:1410.2242 [INSPIRE].

[72] D. Tucker-Smith and N. Weiner, Inelastic dark matter, Phys. Rev. D 64 (2001) 043502 [hep-ph/0101138] [INSPIRE].

[73] D. Tucker-Smith and N. Weiner, The Status of inelastic dark matter, Phys. Rev. D 72 (2005) 063509 [hep-ph/0402065] [INSPIRE].

[74] XENON10 collaboration, J. Angle et al., Constraints on inelastic dark matter from XENON10, Phys. Rev. D 80 (2009) 115005 [arXiv:0910.3698] [InSPIRE].

[75] C. McCabe, The Astrophysical Uncertainties Of Dark Matter Direct Detection Experiments, Phys. Rev. D 82 (2010) 023530 [arXiv: 1005.0579] [INSPIRE].

[76] XENON100 collaboration, E. Aprile et al., Dark Matter Results from 225 Live Days of XENON100 Data, Phys. Rev. Lett. 109 (2012) 181301 [arXiv:1207.5988] [InSPIRE].

[77] LUX collaboration, D.S. Akerib et al., First results from the LUX dark matter experiment at the Sanford Underground Research Facility, Phys. Rev. Lett. 112 (2014) 091303 [arXiv:1310.8214] [INSPIRE].

[78] S. Yellin, Finding an upper limit in the presence of unknown background, Phys. Rev. D 66 (2002) 032005 [physics/0203002] [INSPIRE].

[79] CDMS collaboration, Z. Ahmed et al., Search for inelastic dark matter with the CDMS II experiment, Phys. Rev. D 83 (2011) 112002 [arXiv: 1012.5078] [INSPIRE].

[80] XENON100 collaboration, E. Aprile et al., Implications on Inelastic Dark Matter from 100 Live Days of XENON100 Data, Phys. Rev. D 84 (2011) 061101 [arXiv:1104.3121] [INSPIRE].

[81] DAMA collaboration, R. Bernabei et al., First results from DAMA/LIBRA and the combined results with DAMA/NaI, Eur. Phys. J. C 56 (2008) 333 [arXiv:0804.2741] [INSPIRE].

[82] DAMA and LIBRA collaborations, R. Bernabei et al., New results from DAMA/LIBRA, Eur. Phys. J. C 67 (2010) 39 [arXiv: 1002.1028] [InSPIRE].

[83] R.D. Young and A.W. Thomas, Octet baryon masses and sigma terms from an $\mathrm{SU}(3)$ chiral extrapolation, Phys. Rev. D 81 (2010) 014503 [arXiv:0901.3310] [INSPIRE].

[84] JLQCD collaboration, H. Ohki et al., Nucleon strange quark content from $N_{f}=2+1$ lattice QCD with exact chiral symmetry, Phys. Rev. D 87 (2013) 034509 [arXiv:1208.4185] [INSPIRE].

[85] J. Hisano, K. Ishiwata, N. Nagata and T. Takesako, Direct Detection of Electroweak-Interacting Dark Matter, JHEP 07 (2011) 005 [arXiv: 1104.0228] [INSPIRE].

[86] J. Hisano, K. Ishiwata and N. Nagata, Direct Search of Dark Matter in High-Scale Supersymmetry, Phys. Rev. D 87 (2013) 035020 [arXiv:1210.5985] [INSPIRE].

[87] J. Billard, L. Strigari and E. Figueroa-Feliciano, Implication of neutrino backgrounds on the reach of next generation dark matter direct detection experiments, Phys. Rev. D 89 (2014) 023524 [arXiv: 1307.5458] [inSPIRE].

[88] S.M. Barr and A. Zee, Electric Dipole Moment of the Electron and of the Neutron, Phys. Rev. Lett. 65 (1990) 21 [Erratum ibid. 65 (1990) 2920] [INSPIRE].

[89] D. Chang, W.-F. Chang and W.-Y. Keung, Electric dipole moment in the split supersymmetry models, Phys. Rev. D 71 (2005) 076006 [hep-ph/0503055] [INSPIRE]. 
[90] N.G. Deshpande and J. Jiang, Signals for CP-violation in split supersymmetry, Phys. Lett. B 615 (2005) 111 [hep-ph/0503116] [INSPIRE].

[91] G.F. Giudice and A. Romanino, Electric dipole moments in split supersymmetry, Phys. Lett. B 634 (2006) 307 [hep-ph/0510197] [INSPIRE].

[92] ACME collaboration, J. Baron et al., Order of Magnitude Smaller Limit on the Electric Dipole Moment of the Electron, Science 343 (2014) 269 [arXiv:1310.7534] [INSPIRE].

[93] J.J. Hudson et al., Improved measurement of the shape of the electron, Nature 473 (2011) 493 [INSPIRE].

[94] A.C. Vutha et al., Search for the electric dipole moment of the electron with thorium monoxide, J. Phys. B 43 (2010) 074007 [arXiv:0908.2412] [InSPIRE].

[95] S.D. Thomas and J.D. Wells, Phenomenology of Massive Vectorlike Doublet Leptons, Phys. Rev. Lett. 81 (1998) 34 [hep-ph/9804359] [INSPIRE].

[96] Z. Han, G.D. Kribs, A. Martin and A. Menon, Hunting quasidegenerate Higgsinos, Phys. Rev. D 89 (2014) 075007 [arXiv: 1401.1235] [InSPIRE].

[97] C.H. Chen, M. Drees and J.F. Gunion, Searching for invisible and almost invisible particles at $e^{+} e^{-}$colliders, Phys. Rev. Lett. 76 (1996) 2002 [hep-ph/9512230] [INSPIRE].

[98] OPAL collaboration, G. Abbiendi et al., Search for nearly mass degenerate charginos and neutralinos at LEP, Eur. Phys. J. C 29 (2003) 479 [hep-ex/0210043] [InSPIRE].

[99] C. Hensel, Search for Nearly Mass Degenerate Charginos and Neutralinos in $e^{+} e^{-}$ Collisions, Ph.D. Thesis, Universität Hamburg, Hamburg Germany (2002), http://dx.doi.org/10.3204/DESY-THESIS-2002-047.

[100] H. Baer et al., The International Linear Collider Technical Design Report - Volume 2: Physics, arXiv:1306.6352 [INSPIRE].

[101] M. Berggren et al., Tackling light higgsinos at the ILC, Eur. Phys. J. C 73 (2013) 2660 [arXiv:1307.3566] [INSPIRE].

[102] R. Saito and S. Shirai, Gravitational Wave Probe of High Supersymmetry Breaking Scale, Phys. Lett. B 713 (2012) 237 [arXiv:1201.6589] [INSPIRE].

[103] M. Cirelli, N. Fornengo and A. Strumia, Minimal dark matter, Nucl. Phys. B 753 (2006) 178 [hep-ph/0512090] [INSPIRE].

[104] M. Cirelli and A. Strumia, Minimal Dark Matter: Model and results, New J. Phys. 11 (2009) 105005 [arXiv:0903.3381] [INSPIRE].

[105] J. Hisano, D. Kobayashi, N. Mori and E. Senaha, Effective Interaction of Electroweak-Interacting Dark Matter with Higgs Boson and Its Phenomenology, arXiv:1410.3569 [INSPIRE].

[106] N. Nagata and S. Shirai, Electroweakly-Interacting Dirac Dark Matter, arXiv:1411.0752 [INSPIRE].

[107] T. Takagi, On an algebraic problem related to an analytic theorem of Carathéodory and Fejér and on an allied theorem of Landau, Japan J. Math. 1 (1925) 83.

[108] S.Y. Choi, H.E. Haber, J. Kalinowski and P.M. Zerwas, The Neutralino sector in the U(1)-extended supersymmetric standard model, Nucl. Phys. B 778 (2007) 85 [hep-ph/0612218] [INSPIRE]. 